Published Online on 15 March 2016

Proc Indian Natn Sci Acad 82 No. 1 March 2016 pp. 75-98

(C) Printed in India.

DOI: 10.16943/ptinsa/2016/v82i1/48445

Review Article

\title{
Liquid Crystal Biosensors: New Approaches
}

\author{
SUMYRA SIDIQ and SANTANU KUMAR PAL* iD \\ Department of Chemical Sciences, Indian Institute of Science Education and Research (IISER) Mohali, \\ Sector 81, Knowledge City, Manauli 140 306, India
}

(Received on 12 December 2015; Revised on 13 February 2016; Accepted on 17 February 2016)

\begin{abstract}
This review describes recent advances in the area of research involving the interfacial design of liquid crystal (LC) based biosensors. The first advance revolves around the design and modulation of LC based interfaces for developing LC based stimuli responsive materials. For example, designing nanostructured thin films of LC-based colloidal gels exhibit the sensitivity and specificity with the added benefits of mechanical robustness and processability and can be used to report adsorption of biological and synthetic amphiphiles at aqueous-LC interfaces. In addition, a new pathway for the easy formation of spontaneous uniform LC droplets has been reported that provides a high spatial resolution of micrometers with a very high sensitivity. A second development has focused on the investigations of the ordering of LCs at aqueous interfaces for qualitative and quantitative understanding of important biomolecular interactions for bedside diagnostics and laboratory applications. These important biomolecular interactions include: (i) protein endotoxin interactions as these lead to divergent effects on lipopolysaccharide (LPS)-induced responses, (ii) $\mathrm{pH}$ induced conformation change of cardiolipin (CL) which is known to affect a range of cellular processes and (iii) endotoxin interactions with bacterial cell wall components. Thirdly this study involves design of LC based sensors that hold promise to act as a marker for cells and cell based interactions. Overall, this review illustrates new approaches for developing LC based stimuli responsive materials that are anticipated with fundamental understanding of biomolecular interactions and provide a gateway for further advances involving LCs.
\end{abstract}

Keywords: Liquid Crystals; Biomolecule; Sensing; Aqueous Interface; Ordering Transition

\section{Introduction}

Biomolecular interactions govern the affinity and specificity of complex formation and determine their biological function which could be of enormous scientific and practical importance. The pre-requisite to understand biomolecular function in the context of life and metabolism, it is necessary to analyze interactions of biomolecules by each other. A promising approach to study these interactions is to use liquid crystalline (LC) materials since it is advantageous as many biological systems, including cell membranes, phospholipids, cholesterols, DNA and so forth, exist in LC phases (Stewart, 2003; Steward, 2004). Recently, LCs have been explored for the design of interfaces that mediate desired interactions with biological systems (Lowe and Abbott, 2012). LC materials allow label-free observations of biological

\footnotetext{
*Authorfor Correspondence: E-mail: skpal@iisermohali.ac.in
}

phenomena as the orientational properties of LCs enable the amplification and the transduction of biologically relevant binding events at nanostructured surfaces into optical outputs visible by naked eye (Brake et al., 2003a; Lin et al., 2011; Brake and Abbott, 2002; Brake et al., 2003b; Lockwood et al., 2005; Price and Schwartz, 2008; Brake et al., 2005; Lockwood et al., 2006; Sivakumar et al., 2009; Gupta et al., 2009; Park and Abbott, 2008; Hu and Jang, 2012; Bai et al., 2014; Khan and Park, 2014; Sadati et al., 2015; Zhu and Yang, 2015; Wang et al., 2015; Munir et al., 2015). The study of biomolecular interaction using $\mathrm{LC}$ material relies on the interaction between the sensing LC and the biomolecule of interest. However, realization of this potential requires advances in the interfacial design of LC materials, which is the subject of our main focus. 
The most common LC phase is the nematic phase of the class of thermotropic LCs. (Thermotropic LCs exhibit changes in phases as a function of temperature). A nematic phase exhibits an orientational order, but lacks positional order (Fig. 1A). The orientational order of mesogens within a nematic can be described by the statistical preference of the mesogens to align in one direction (de Gennes and Prost, 1994; Collings and Hird, 1997). Unlike isotropic liquids, this orientational preference is typically controlled by interfacial energetics on the scale of $10^{-3}$ to $1 \mathrm{~mJ} / \mathrm{m}^{2}$ (Cadwell et al., 2006; Clare et al., 2006; Jerome, 1995) and communicated over distances much greater than the size of the mesogens $(\sim 100$ $\mu \mathrm{m}$ is typical for a nematic LC) (Fig. 1B). The longrange orientational ordering of molecules within the nematic LC also leads to anisotropic optical properties that enable characterization of the orientational order via the use of polarized light. Inspired by the sensitivity of the orientational order of LCs to the chemical functionality and organization of molecules at interfaces a wide range of amphiphiles (Carlton et al., 2013), such as phospholipids (Brake et al., 2003a; Brake et al., 2005; Lockwood et al., 2006) and surfactants (Brake and Abbott, 2002) at aqueous-LC interfaces can be reported through changes in the optical appearance of the LCs. Brake et al., (2003)

\section{A Crystal}

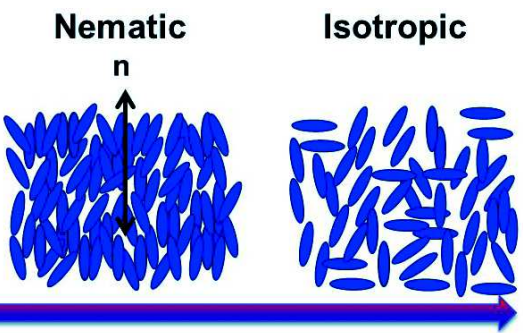

Increasing temperature

B

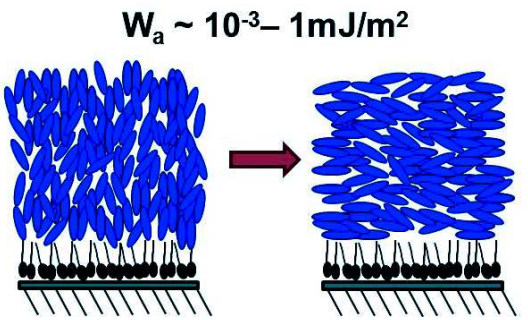

Fig. 1: A) Schematic illustration of the nematic LC phase has an orientational order, but lacks long-range positional order, and exists between the crystalline and isotropic phases. $n$ is the nematic director. B) Surface sensitive orientational ordering of LCs controlled by interfacial energetics reported that these changes in optical appearance result from strong coupling between aliphatic tails of the adsorbed amphiphiles and the mesogens of the LC. The advantages of these adsorbed surfaces make them attractive for a number of potential applications involving more complex interfacial phenomena, such as high-throughput screening of enzymatic activity (Park and Abbott, 2008) and molecular binding events (Hu and Jang, 2012; Hartono et al., 2009; Tan et al., 2012; Liu et al., 2013; Hu and Jang, 2012) through LC reorientations driven by chemical or physical events that perturb the organization of the monolayer interfaces. Enthused by the budding utility of LC materials in biological applications (particularly, reporting biological interactions), in this paper we report progress made in our laboratory toward the design of interfaces of LC materials such that desired interactions are realized between the LC materials and biological systems. The advances of our research described here focus on the design and modulation of LC based interfaces based on stimuli responsive materials. The study also advances the use of LC based aqueous interfaces for qualitative and quantitative understanding of important biomolecular interactions for bedside diagnostics and laboratory applications. Finally, we have shown the direct protein LC interactions in aqueous environment which would fulfill significant knowledge gap in understanding the intermolecular interactions that underlie the direct ordering of LCs with protein-decorated surfaces and hold great promise to provide LC as biosensors for cell-surface interface.

\section{Approaches for Designing Liquid Crystal Based Sensors for Biological Applications}

\section{Colloidal Liquid Crystal Gels for Biomolecular Amplification}

Building from past studies, herein we report that it is possible to prepare thin films of LC-based colloidal gels that are stable under water and can be used to report adsorption of biological and synthetic amphiphiles at LC interfaces. In particular, we have found that the sensitivity can be improved by forming a gel which is highly stable and retains its structure on one of the confining surfaces, or when the supporting surface was immersed into water. Unlike LCs within TEM grids, these CLC gels are selfsupporting, mechanically strong and can be easily 
handled. This study has been motivated by the goal of designing nanostructured LC materials that exhibit the sensitivity and specificity with the added benefits of mechanical robustness and processability. The micro-structured colloidal liquid crystal (CLC) gels described in this study are based on the suspensions of colloids in low molecular weight E7 nematic LC. E7 is a mixture of four alkoxycyanobiphenyls ( $n \mathrm{CBs}$ ) with different aliphatic chain length: $51 \mathrm{wt} . \%$ 4-cyano4'-n-pentyl-biphenyl (5CB), 25 wt.\% 4-cyano-4'- $n$ heptyl-biphenyl (7CB), 16 wt.\% 4-cyano-4'-noxyoctyl-biphenyl (8OCB) and 8 wt.\% 4-cyano-4'n-pentyl-p-terphenyl (5CT). Agarwal et al., (2008) and Pal et al., (2009) demonstrated that when colloidal suspensions of microspheres are prepared in the isotropic phase of LCs followed by cooling below their isotropic-nematic transition temperature $\left(\mathrm{T}_{\mathrm{NI}}\right)$, they form birefringent waxy semi-solid (gel) with significant storage modulus $\left(\mathrm{G}^{\prime} \sim 10^{3}-10^{5} \mathrm{KPa}\right)$. This gelation has been suggested to result from the formation of a particle network resulted from expulsion of colloids from the nematic LC as it cools down from isotropic to nematic phase. It is driven by orientational elasticity of the nematic phase and the anchoring energy and surface tension at LC/particle interface; however, the mechanism is not fully understood. In this study, we have shown that it is possible to use organized networks of micrometer sized particles to form LC containing composite materials that would hold its structure at aqueous/LC gel interface and also retain their responsiveness to changes in chemical environment at those interfaces (Agarwal et al., 2013). Although, previous reports have demonstrated that self-assembly of amphiphilic molecules at aqueous/ LC interfaces can trigger ordering transitions in LCs such as 5CB, 5CB droplets, the influence of colloidal network within the LC gel in the surface-driven ordering transition has not been previously demonstrated (Lin et al., 2011; Gupta et al., 2009; Clegg et al., 2003; Tong et al., 2009; Wood et al., 2011).

We report that the thin films of CLC gels (thicknesses of $\sim \mu \mathrm{m}$ ) confined between chemically functionalized glass slides such that nematic domains, confined by colloidal network, extend across the entire thickness of thin films (Fig. 2). As the LC in the E7 nematic domains $(\sim 6-10 \mu \mathrm{m})$ of these thin films interacts with the confining surfaces on either side,

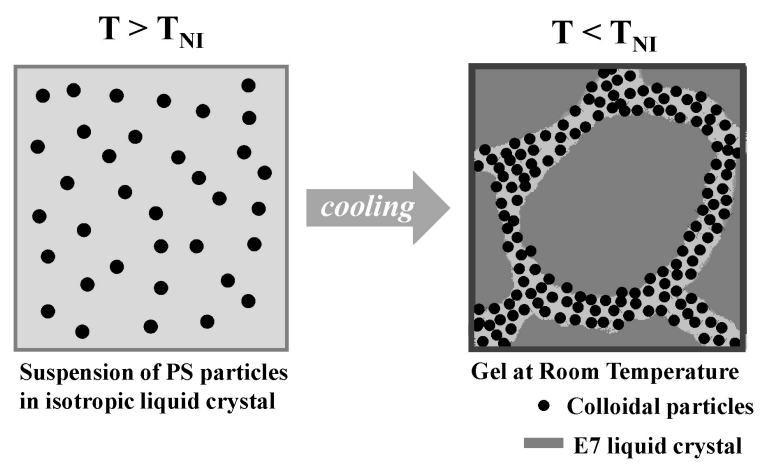

Fig. 2: Schematic illustration of the thin films of CLC gels formed between glass slides. (Redrawn with permission from Agarwal et al., (2013))

the ordering of LC in the domains could be influenced by the chemistry of the confining surfaces. Fig. 3 shows polarized light and bright-field micrographs of a thin film of CLC gel supported on a glass microscope slide treated with $N, N$-dimethyl- $n$-octadecyl-3aminopropyltrimethoxysilyl chloride (DMOAP) which anchors the LC at the LC-glass interface in a homeotropic orientation. We note that the polarized optical micrograph reveals the dark optical appearance of the CLC gel in Fig. 3A, consistent with nearuniform homeotropic orientation of LC within the CLC gel whereas bright field micrograph reveals the CLC domain structure. Next, the thin film of CLC gel supported on the DMOAP-treated glass immersed under water determines whether CLC gels would dewet the surface. Inspection of the polarized light micrograph (Fig. 3A) of the CLC film upon immersion in water revealed bright optical appearance resulting that the domain structure of the CLC gel is not perturbed/destroyed and remain stable under water. The bright optical appearance of the CLC gel in Fig. $3 \mathrm{~B}$ is consistent with a change from homeotropic anchoring when the free interface of the CLC gel was in contact with air to a planar orientation after immersion under water (see Fig. 3E for a schematic illustration).

To the aqueous solution covering the CLC gel, we next added the biological amphiphile lipopolysaccharide (LPS). Also called endotoxin, found in the outer membrane of gram-negative bacteria, comprises a polysaccharide head group and a lipid with 6 tails (called lipid A) which is responsible for its toxicity. Inspection of Fig. 3C reveals that following the addition of $20 \mu \mathrm{g} / \mathrm{mL}$ of LPS to the aqueous solution 
in contact with the CLC gel, the CLC gel remained stable and the optical appearance of the CLC gel changed from bright to dark. The change in optical appearance of the CLC gel is indeed consistent with an adsorbate-driven anchoring transition of the LC (to a homeotropic orientation) at the LC-aqueous interface of the LC rich domains of the gel (Fig. 3C). This result is consistent with Lin et al., (2011) report of homeotropic anchoring of nematic LCs at LPSdecorated interfaces. Quantification of the intensity of light transmitted through a macroscopic region of the CLC gel, as shown in Fig. 3G, reveals that the anchoring transition occurred over approximately 15 min following the addition of the LPS. The careful analysis of time-lapse images of the CLC gel revealed that the LC domains that possessed a dark optical appearance prior to contact with LPS, underwent a transient change in optical appearance and birefringence upon contact with the LPS solution. This result supports our conclusion that these domains appeared dark prior to contact with LPS because the azimuthal orientation of the LC within the domains was aligned with one of the polars. An analysis of the change in optical appearance of individual domains of LC within the gel, leads us to conclude that, at the single-domain level, the LPS-triggered change in optical appearance of the LC differed substantially from one domain to the next. This heterogeneity in the response of the domains likely reflects variation in the sizes and shapes of the LC-rich domains in the gel, and thus differences in elastic and surface anchoring energies for each domain. It also suggests that manipulation of the size and shape of the LC domains may provide a means to rationally tune the response of CLC gels to interfacial adsorbates. In addition to biological lipids such as LPS, synthetic surfactants (e.g., sodium-dodecyl sulfate (SDS)) also trigger anchoring transitions in the LC-rich domains of the CLC films.

Finally, we demonstrate that CLC gels can be used to report the activity of enzymes, when the substrates for the enzymes decorate the aqueous interface of the CLC gel. Inspection of Fig. 4A shows that the LC-rich domains within the CLC gel undergo an anchoring transition in the presence of ${ }_{\mathrm{L}}$-DLPC, consistent with formation of a monolayer of ${ }_{L}$-DLPC at the aqueous interface of the LC as reported by Brake et al., (2003a) (and thus homeotropic anchoring, Fig. 4B). Next, we removed the residual ${ }_{\mathrm{L}}$-DLPC from the aqueous solution and introduced the enzyme phospholipase $\mathrm{A}_{2}\left(\mathrm{PLA}_{2}\right)$. PLA $\mathrm{A}_{2}$ is known
A

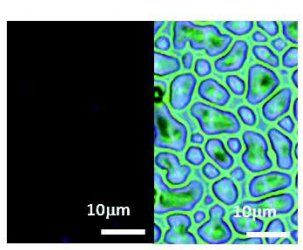

B

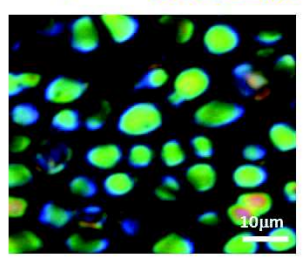

C

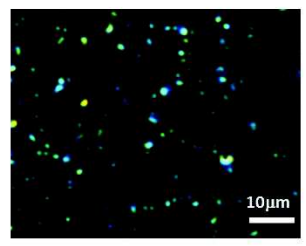

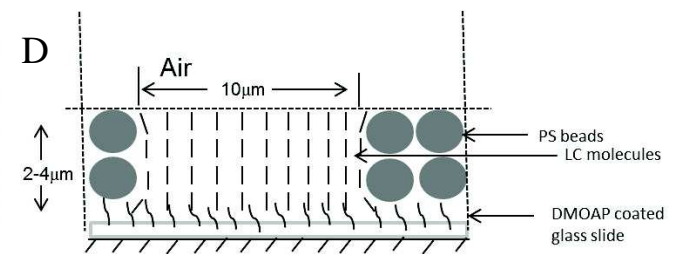
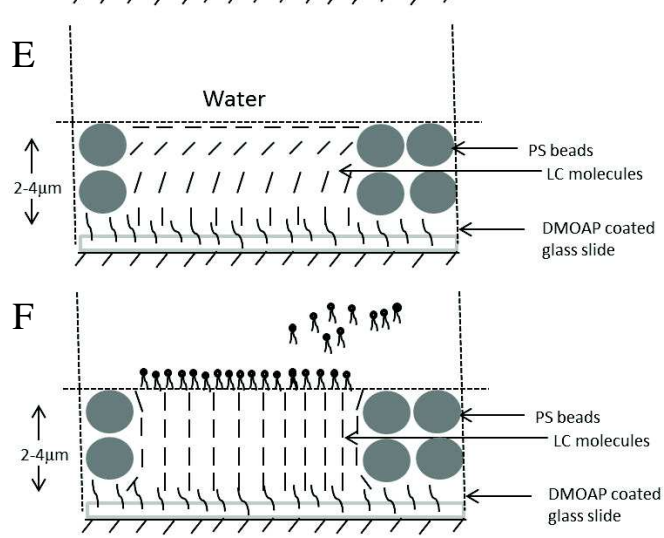

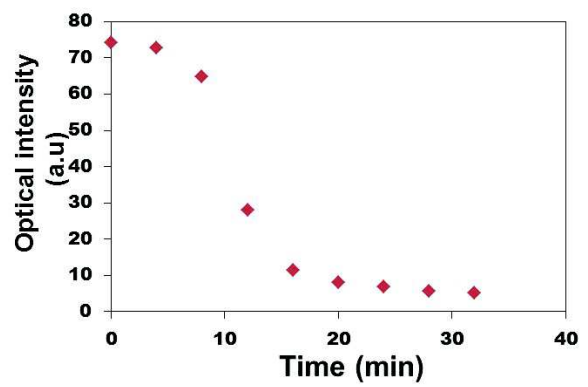

Fig. 3: Optical micrograph of $15 \%$ E7 gel (conc.: $0.03 \mathrm{mg} / \mathrm{mL}$; thickness: $2-4 \mu \mathrm{m}$; scale bar: $10 \mu \mathrm{m})$ (A) in contact with DMOAPcoated glass slides (B) upon addition of water (C) upon addition of LPS. (D-F) Corresponding schematic illustration of CLC gels exposed to aqueous LPS solution. (G) Optical intensity of the time-lapse polarized-light images of the thin film of CLC-gel cast on a DMOAP-treated glass slide following contact with $20 \mu \mathrm{g} / \mathrm{mL}$ aqueous LPS. (Reproduced with permission from Agarwal et al., (2013)) 
to catalyze the hydrolysis of ${ }_{\mathrm{L}}$-DLPC in the presence of $\mathrm{Ca}^{2+}$, thus resulting in the formation of the lysophospholipid and the corresponding fatty acid. As shown in Fig. 4C, during the $1 \mathrm{~h}$ period following the addition of $10 \mathrm{nM}$ of $\mathrm{PLA}_{2}$ into the aqueous phase, the LC rich domains of CLC gel were observed to undergo an ordering transition. The change in optical appearance of the gel from dark to bright over a period of $60 \mathrm{~min}$ is consistent with Brake et al., (2003a) studies of hydrolysis of ${ }_{\mathrm{L}}$-DLPC by $\mathrm{PLA}_{2}$ (the anchoring transition is caused by desorption of the hydrolysis products of the enzymatic reaction from the interface). This observation confirms that LCrich domains of CLC films can be driven through reversible anchoring transitions via the introduction and removal of adsorbates at the aqueous-CLC gel interface. We also note that the overall rate of response of the E7 to enzymatic activity of PLA $\mathrm{A}_{2}$ is greater when the E7 is hosted within a CLC gel as opposed to a TEM grid. This difference, as noted above, is likely related to the difference in the thickness of the LC in the domains of the CLC gel and the TEM grid, and thus the influence of the elastic energy of the LC on the orientation of the LC. Overall, we conclude that the CLC gels offer a general and facile approach to the preparation of "free interfaces" of LCs suitable for design of LC-based stimuli-responsive materials.

\section{A New Pathway for the Formation of Liquid Crystal Droplets within Confined Boundaries}

The second approach towards the design of LC materials for biological applications revolves around the new way of preparing LC droplets. The approach was motivated by the observation that LC droplets have been widely appreciated as a new class of functional materials due to their large surface areas, rich phases, well-defined director configurations and unique tunable optical properties (Lin et al., 2011;

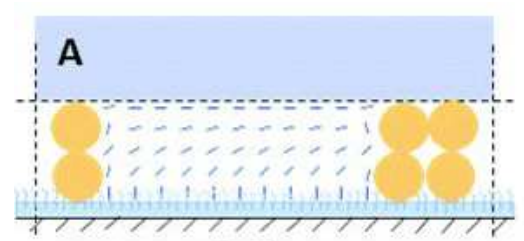

B $\int L-D L P C$
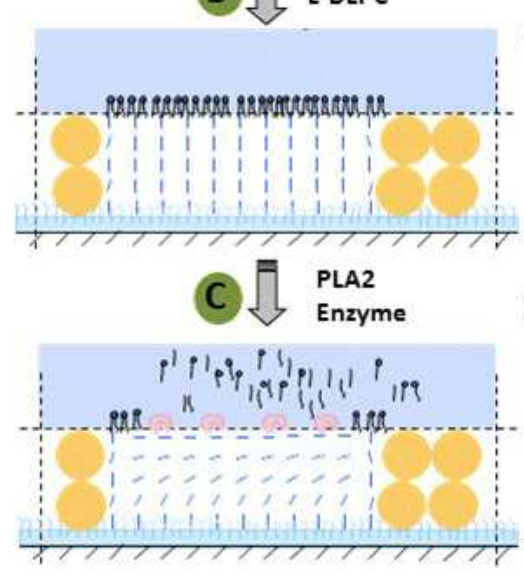


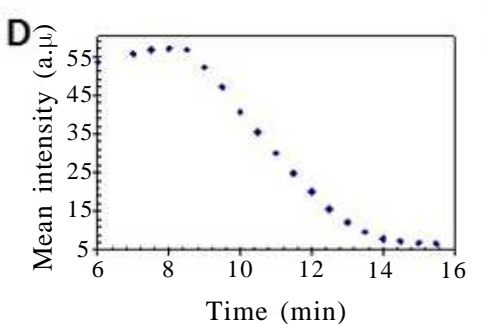

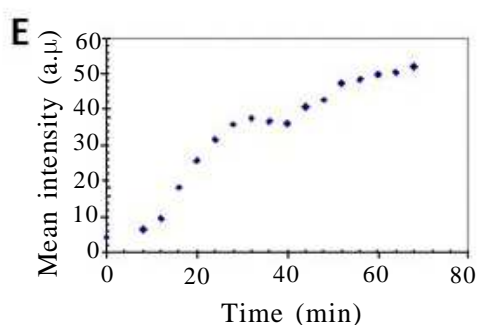

Fig. 4: A) Schematic representation of the expected orientational change of LC molecules confined in LC-rich domains of CLC gels exposed to aqueous ${ }_{L}$-DLPC solution followed by PLA $A_{2}$ enzyme treatment. B) Time-lapse polarized light micrographs of a thin CLC gel film cast on an OTS-treated glass slide following exposure to $20 \mu \mathrm{g} / \mathrm{mL} \mathrm{L}_{\mathrm{L}}$-DLPC. The change in optical intensity of the polarized-light images of the CLC-gel films as a function of time following incubation with aqueous $20 \mu \mathrm{g} / \mathrm{mL}_{L}$-DLPC is plotted in the graph. C) Optical micrographs (between crossed-polars) of a CLC gel thin film on OTS-functionalized glass, with ${ }_{L}$-DLPC absorbed on the aqueous/LC surface, at successive time points after incubating against $10 \mathrm{nM} \mathrm{PLA} \mathbf{P}_{2}$. The change in optical intensity of the polarized-light images of the CLC-gel films as a function of time following incubation with aqueous $10 \mathrm{nM} \mathrm{PLA}$ is plotted in the graph. $D$, E) The measured intensity of light transmitted through the CLC gel (crossed polars), corresponding to images (A) and (B), respectively. (Reproduced with permission from Agarwal et al., (2013)) 

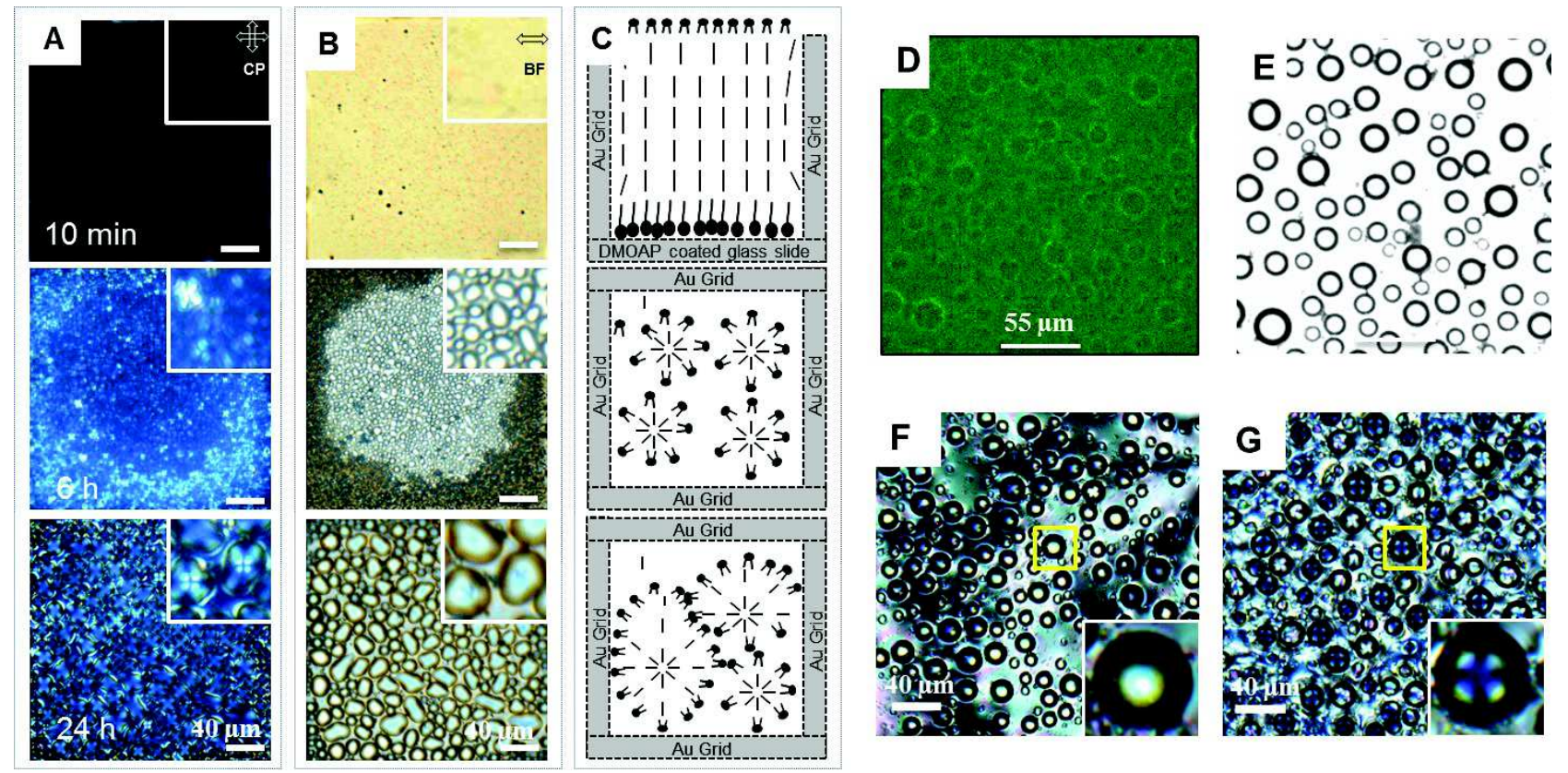

Fig. 5: A) Crossed polars (CP) and (B) bright field (BF) images of aqueous-5CB interfaces within the TEM grids supported on DMOAP-coated glass slides upon exposure to $0.5 \mathrm{mg} \mathrm{mL}^{-1}$ phosphatidyl choline (PC) after $10 \mathrm{~min}, 6 \mathrm{~h}$ and $24 \mathrm{~h}$. The insets (in (A) and (B)) show the corresponding high-magnification images that reveal the formation of stable and welldeveloped LC droplets exhibiting radial configuration. (C) Top to bottom: schematic illustration of the time dependent formation of LC droplets with radial LC ordering. (D) Fluorescence micrograph and (E) the corresponding bright field image of nematic 5CB hosted within a gold grid supported on a DMOAP-treated glass substrate after contact with PC doped with $1 \mu \mathrm{M}$ fluorescent PC (NBD PC) for 3 days. (F) Polarized optical micrograph (crossed polars) of nematic 5CB hosted in a gold grid supported on a DMOAP-treated glass substrate after contact with water for 3 days. Water droplets formed spontaneously on the LC film. (G) Polarized optical micrograph of the aqueous-5CB system (as in (F)) on exchanging the aqueous phase with the $P C$ solution after $19 \mathrm{~h}$. The well-developed water droplets reorganize to LC droplets with radial LC ordering. (Reproduced with permission from Sidiq et al., (2014))

Sivakumar et al., 2009; Alino et al., 2011; Alino et al., 2012; Bera and Fang, 2012; Bera and Fang, 2013; Gupta et al., 2009; Khan et al., 2011; Kinsinger et al., 2010; Zou et al., 2011). In particular, these offer routes to design simple, economic and convenient passive sensing devices that provide a high spatial resolution of micrometers with a very high sensitivity. We report a new pathway for the easy formation of spontaneous uniform LC droplets. While the techniques reported earlier (Miller and Abbott, 2013) have resulted in the preparation of LC droplets with a life span of a few hours, our approach has provided LC droplets with a stability of days to months (Sidiq et al., 2014).

For this, we incubated 5CB in the pores of 20 $\mu \mathrm{m}$ thick electron microscopy grids supported on DMOAP-coated glass slides in an aqueous solution of phosphatidyl choline (PC) $\left(0.5 \mathrm{mg} \mathrm{mL}^{-1}\right)$ for $3 \mathrm{~h}$.
Inspection of Fig. 5A revealed that the dark optical appearance (homeotropic anchoring) of the LC immediately due to the adsorption of PC to an aqueous-5CB interface. After incubation for $3 \mathrm{~h}$ or more, we observed well-developed droplets which were characterized by a single point defect located at the center of the droplets shown in the bright field optical micrograph image (Fig. 5B). In order to provide a further insight into the microstructure of the LC droplets in the presence of PC, fluorescently tagged $\mathrm{PC}$ was used to examine the location of the surfactant (PC) in the co-assembly. Inspection of the combined bright and epifluorescence micrographs (Fig. 5D and $5 \mathrm{E})$ revealed that the fluorescence was mainly present at the boundary of (and also in between) the droplets, with no fluorescence at the center of the droplets. This led us to conclude that the formation of the PC monolayer stabilizes these droplets by rearranging itself around the droplets. 
It has been reported (Yang and Abbott, 2010) that a micrometer-thick film of the LC supported on OTS (octadecyltrichlorosilane) treated glass substrates led to the formation of water droplets when immersed under water. To provide an insight into whether PC is responsible for the formation of $\mathrm{LC}$ droplets with radial LC ordering, we incubated a LC film (supported on DMOAP-coated glass slides) in an aqueous phase for three days followed by an exchange of the aqueous phase with the PC solution. Interestingly, when exchanging the aqueous phase with the $\mathrm{PC}$ solution we observed a topological defect formation within the droplet after $19 \mathrm{~h}$ (Fig. 5F and 5G). We note two observations from this study. First, the regions of LCs around the droplets have transformed into a homeotropic orientation, as evidenced by the black areas. Second, LC droplets with topological defects are formed in the same location where water droplets were previously present. We do not yet fully understand the reason for the formation of LC droplets from water droplets after adding PC. It may be hypothesized that in the presence of PC, there could be a gradual replacement of water by LCs which is stabilized through topological defects.

The combination of the results described above leads to the following overall understanding of the LC droplet formation. During the formation of the macro emulsion, the reduction of the interfacial tension due to the use of PC reduces the amount of mechanical work required to break the inner phase into dispersed particles. It has been reported (Lavrentovich, 1998) that the formation of an equilibrium state of a LC droplet which could be described as the minimum of the free energy functional $(F)$, composed of both a volume $\left(F_{v}\right)$ and a surface part $\left(\mathrm{F}_{\mathrm{s}}\right)$. The $\mathrm{F}_{\mathrm{s}}$ can again be modeled as surface tension which has an isotropic part $\sigma$ and an anisotropic part $\mathrm{W}_{\mathrm{A}}$ (i.e., $\mathrm{Fs} \sim 4 \pi\left(\sigma \mathrm{r}^{2}+\mathrm{W}_{\mathrm{A}} \mathrm{r}^{2}\right), \mathrm{r}$ being the radius of the droplet). In the case of cyanobiphenyl, when the anchoring part $\mathrm{W}_{\mathrm{A}}\left(10^{-5}\right.$ to $\left.10^{-6} \mathrm{~J} \mathrm{~m}^{-2}\right)$ is much smaller than $\sigma\left(10^{-3}\right.$ to $\left.10^{-2} \mathrm{~J} \mathrm{~m}^{-2}\right)$, $\mathrm{F}_{\mathrm{s}}\left(4 \pi \sigma \mathrm{r}^{2}\right)$ is dominated by the surface tension and thus proportional to $\mathrm{r}^{2}$. It has been demonstrated by Harrison and Fisch, (2000) that PC could decreases from $10^{-3}$ to $10^{-4} \mathrm{~J} \mathrm{~m}^{-2}$ which in turn decreases $\mathrm{F}_{\mathrm{s}}$. In such cases, $\mathrm{F}$ is reduced to a greater extent which results in the formation of LC droplets, stabilized by topological defects. In addition to the reduction of the interfacial tension, PC as reported by Prischepa et al. (2005) imposes the anchoring boundary conditions for the nematic director. $\mathrm{PC}$, due to its hydrophobic part, interacts with the alkyl chain of $5 \mathrm{CB}$ and results in the homeotropic ordering of the LC. It then rearranges itself around the LC molecules and creates a radial arrangement around the $\mathrm{LC}$ molecules where each droplet has a single point defect at the center (called hedgehog). It has been demonstrated (Terentjev, 1995) that the stability of the nematic macro emulsions is greatly enhanced due to the energy barrier created by the elastic constant of the LC and surface tension is very high $\left(\sim 10^{-17} \mathrm{~J}\right)$ compared to the typical thermal energy at room temperature. Hence, it is very likely that in the presence of PC, there is an additional contribution of the elastic energy to the formation of the topological defects in the confined nematic and radial LC droplets, which prevents coagulation compared to their isotropic counterparts.

We investigated and compared the stability with respect to time and size distribution of the LC droplets to those of existing techniques. It has been found (Miller and Abbott, 2013) that the techniques reported for the preparation of LC droplets have a life span of only a few hours whereas the droplets prepared by our method are quite stable for a period of several days. This is because in our case, the droplets formed in a confined boundary created by the grid system and thus stabilized (less mobile). In addition, the LC droplets (with radial configuration) were quite stable and reversible i.e., the optical responses remain unchanged by the temperature-induced phase transition of the LCs. These experiments also confirm the suitability of our approach towards a simple and robust platform using LC droplets for further applications.

Next, we reported the role of other surfactants and lipids and explored their behavior on incubation at aqueous-5CB interfaces. We have chosen SDS, CTAB, lysophosphatidic acid (LPA), 1,2-dilauroyl-snglycero-3-phosphocholine (DLPC) and LPS and studied their behavior at aqueous-5CB interfaces. It has found that both CTAB and DLPC resulted in the formation of nice and well developed droplets with radial LC ordering. Second, LPA does not lead to the formation of stable droplets whereas no droplet 
formation was observed in the presence of LPS and SDS. These results reveal for the first time that, in addition to inducing homeotropic alignment of the LC, the ionic charge in the lipids can play an important role for the spontaneous formation of LC droplets.

Motivated by the above observations, we investigated the topological ordering of LCs within droplets to report interfacial enzymatic reactions. It was reported (Gupta et al., 2009) that PLA triggers a transformation of the LC droplet from a radial configuration to a bipolar configuration decorated with ${ }_{\mathrm{L}}$-DLPC. Interestingly, inspection of the polarized light micrograph of the LC droplets in response to the adsorption of $\mathrm{PLA}_{2}(150 \mathrm{nM})$, an anchoring transition of the LC was observed from an initially homeotropic orientation (radial configuration in the presence of PC) to a planar orientation with a bipolar topological defect (Fig. 6A). These bipolar droplets were then further explored in detecting various bio-molecules such as bacterial phospholipids (LPS). We investigated this phenomenon with LC droplets formed through enzymatic degradation of PLA $_{2}$ (bipolar) and observed a structural transformation to radial configuration (Fig. 6B). The optical response of these LC droplets with a bipolar configuration to radial configuration was observed after the adsorption of LPS. This result, we
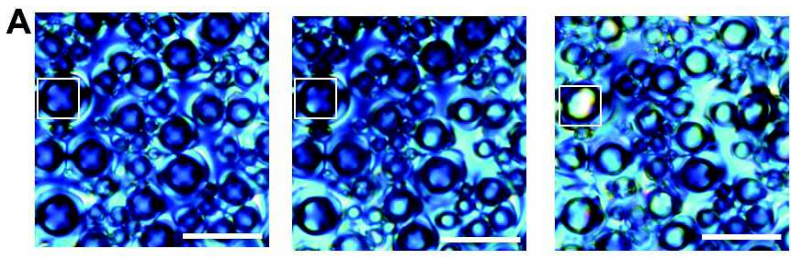

B
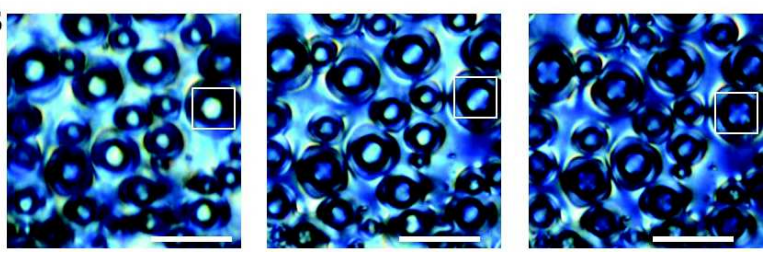

Fig. 6: A) Optical images (crossed polars) of 5CB droplets covered with DLPC upon exposure of $150 \mathrm{nM}^{\mathrm{PLA}}$ into an aqueous solution of $\mathrm{TBS}(\mathrm{pH}=8.9)$ containing $10 \mathrm{mM} \mathrm{CaCl}_{2}$ indicating the change in the anchoring transition of the LC droplets from radial to bipolar upon adsorption of $\mathrm{PLA}_{2}$. B) Represents the time lapse polarized micrographs of aqueous-5CB interface following contact with $100 \mathrm{mg} / \mathrm{mL}$ aqueous LPS and changed the anchoring transition of LC droplets from bipolar to radial upon adsorption of LPS. Scale bar = $40 \mu \mathrm{m}$. (Reproduced with permission from Sidiq et al., (2014)) believe in principle, enables new pathways to exploit interfacial adsorbate-induced properties of LC droplets.

\section{Understanding the Important Biomolecular Phenomenon using Liquid Crystals}

\section{A Simple Quantitative Method to Study Protein- lipid Interactions using Liquid Crystals}

It is important to understand the interaction of proteins with endotoxins as it led to divergent effects on LPSinduced responses (Tesh and Morrison, 1988; Roth and Kaca, 1994; Machnicki et al., 1993; Yu and Wright 1996; Brandenburg et al., 1998), which serve as a basis for many clinical and therapeutic applications. Inspired by this, we report a simple but useful advance in the design of an experimental system that enables a quick, quantitative, and label-free detection of LPSbiomolecular interactions (Das et al., 2015). Our approach revolves around surface-driven ordering transitions in LCs to quantify the interaction between LPS and biomolecules at aqueous-LC interfaces in a planar geometry. There are a number of biomolecules, namely, bovine serum albumin (BSA), lysozyme (LZM) and hemoglobin ( $\mathrm{Hb})$, that show diverse interactions with endotoxins. These three proteins of different categories not only possess different charges at physiological $\mathrm{pH}$, but also have diverse effects on endotoxicity for interaction with LPS (Jurgens et al., 2001; Takayama et al., 1990; Ohno and Morrison, 1989). Yang and Yang, (2008) demonstrated that these proteins had different affinities towards LPS, which led to different kinetic parameters being measured by a surface plasmon resonance (SPR) biosensor.

To study the interactions between LPS with these proteins, we first studied the optical response of LCs coupled to the negatively charged LPS at aqueous/LC interfaces. As already reported by Lin et al. (2011) that the self-assembly of LPS molecules at aqueous/LC interfaces trigger ordering transitions in LCs; thus leading to homeotropic anchoring of LCs. Motivated by this, we hypothesized that the addition of proteins at these interfaces switch the orientational ordering of LCs and thus, demonstrate the promise of LC-aqueous interfaces for reporting interfacial phenomena (see schematic Fig. 7A). To determine the influence of these proteins (described above) on the orientational ordering of 5CB within LPS-laden 
aqueous-LC interfaces, we exposed an aqueous solution of $10 \mu \mathrm{M} \mathrm{Hb}$ in Tris buffer ( $\mathrm{pH}$ 7.4) to the LPS-laden aqueous/5CB interface. An immediate change in the optical appearance of the LC from dark (homeotropic orientation) to bright (planar/tilted orientation) was observed within $15 \mathrm{~s}$ of the addition of $\mathrm{Hb}$ (Fig. 7B and 7C). The planar/tilted orientation of the LCs, when combined with prior reports of biomolecular interactions in LCs, is suggestive of a physical phenomenon, whereby $\mathrm{Hb}$ absorbs and organizes itself at the LPS aqueous-LC interface. This observation could be due to strong hydrophobic interactions between neutral $\mathrm{Hb}$ and negatively charged LPS molecules (Jurgens et al., 2001), which play an important role in the rearrangement of the LPS monolayer and thus result in a transition in the orientation of LCs from dark to bright. Our next goal was to investigate whether the interaction of negatively charged BSA with negatively charged LPS membrane at the aqueous-LC interface underwent a similar ordering transition to that of LPS-Hb described above. Thus, $10 \mu \mathrm{M}$ BSA in Tris buffer (pH 7.4) was added to the aqueous-5CB interface decorated with LPS. We observed a rapid change in the optical appearance of the LCs from dark to bright (Fig. 7D and 7E). The change in the orientation of the LCs from homeotropic to planar indicates that the LPS interaction with serum proteins is driven by hydrophobic interactions and can be studied at the LC/aqueous interface. Likewise, in response to the LZM protein, which formed nonspecific interactions with LPS, a similar change was observed in the optical appearance of the LCs

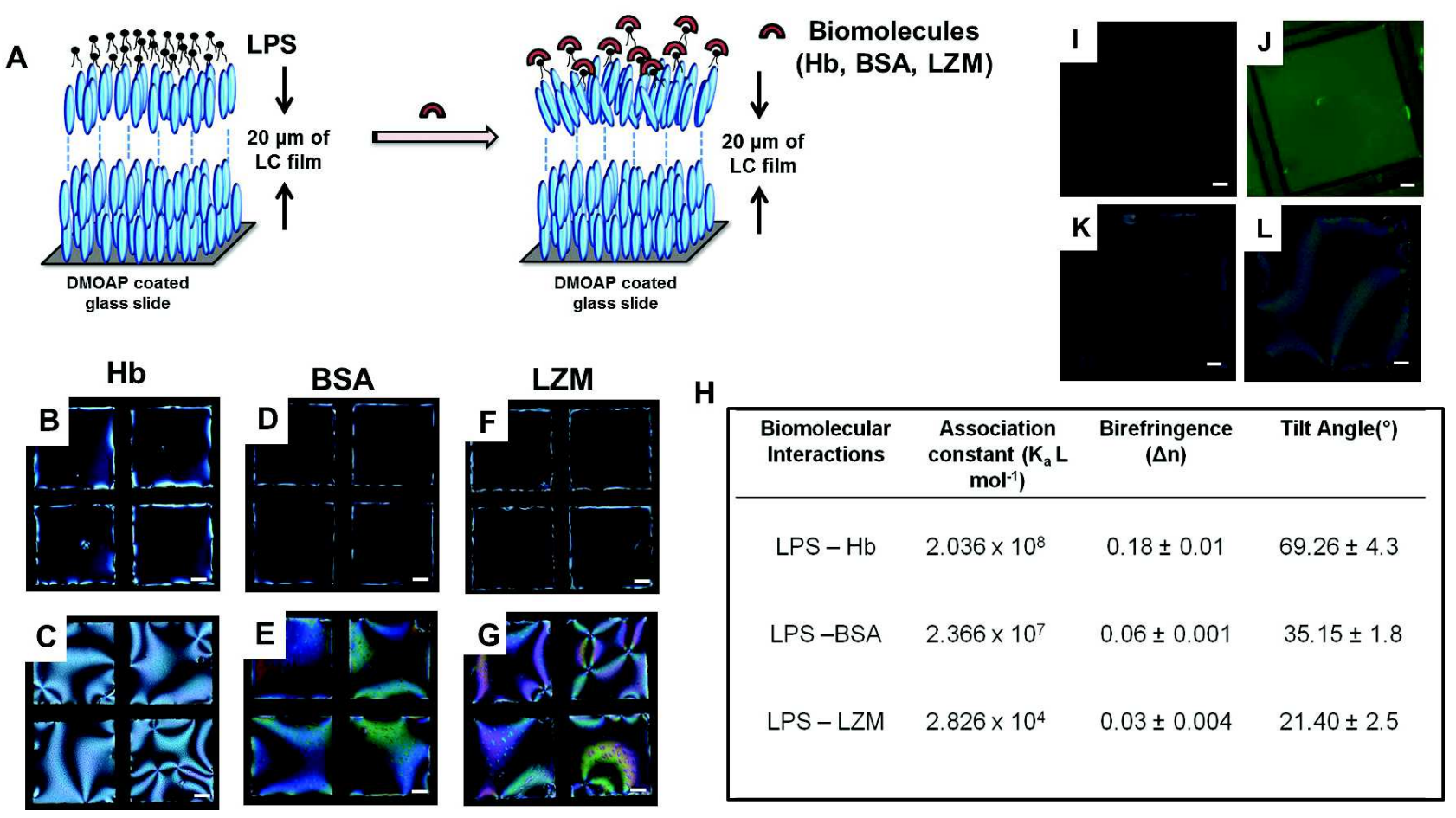

Fig. 7: A) Schematic illustration of the orientation of the 5CB LC coupled to the addition of biomolecules (Hb, BSA and LZM) onto the negatively charged LPS-decorated interface. Orientation of $5 \mathrm{CB}$ on the LPS-laden aqueous-LC interface leading to homeotropic anchoring and the LPS-laden aqueous-LC interface exposed to biomolecules, leading to planar anchoring. B), D), F) Optical images (crossed polarizers) of 5CB hosted in gold grids supported on DMOAP-coated glass slides and placed into contact with an aqueous solution of Tris buffer, followed by the introduction of LPS, and incubation for $2 \mathrm{~h}$. Slides after contacting the LPS laden interface with $10 \mu \mathrm{M}$ aqueous solutions of Hb (C), BSA (E) and LZM (G). Scale bar $=40 \mu \mathrm{m}$. H) Table representing the correlation of the binding constants of interactions between LPS and proteins along with tilt angles and birefringence values of $5 \mathrm{CB}$. Epifluorescence micrographs of LPS laden aqueous-5CB interface (containing $100 \mathrm{nM}$ BSA and $20 \mathrm{nM}$ BSA-FITC) following 90 min incubation against (I) an aqueous buffer of pH 7.4 (10 mMTris) and $(\mathrm{J})$ an aqueous solution of BSA. Corresponding micrographs (crossed polarizers) of the nematic 5CB film are shown in $\mathrm{K}$ ) and $\mathrm{L}$ ) respectively. (Reproduced with permission from Das et al., (2015)) 
from dark to bright after immersion of the LPS-laden 5CB interface into a $10 \mu \mathrm{M}$ aqueous solution of LZM in Tris buffer at pH 7.4 (Fig. 7F and 7G). These measurements revealed that the LPS-laden aqueousLC interface could provide the basis for a general, facile method to tune and possibly quantify the response of different proteins. It is noteworthy, however, that in all cases the dynamic ordering transitions towards a planar ordering in different $\mathrm{pH}$ conditions other than physiological $\mathrm{pH}$ were the same, which indicated that the ordering of the LC was independent of electrostatic interactions. This also suggests that specific and nonspecific interactions of different types of protein with endotoxins, leading to changes in the orientation and optical appearance of LCs, can be attributed to changes in the ordering of LPS molecules at the interface through hydrophobic interactions.

Since different proteins have different binding affinities towards LPS, so we hypothesized that the extent of disruption of a self-assembled monolayer of LPS depends upon the strength of binding of proteins with LPS, leading to different tilts of LC molecules. Therefore, measurement of the tilt angle, by quantifying the retardation of the LCs by using a birefringence mapping system, might provide additional insight into the physical phenomena that underlie LPSprotein binding events driven by the ordering transition of LCs at aqueous-LC interfaces. For this, we measured the effective birefringence of $5 \mathrm{CB}$ immediately after contact with $50 \mathrm{nM}$ of each protein ( $\mathrm{Hb}, \mathrm{BSA}$ and LZM) at the LPS-aqueous-LC interface. After calculating the tilt angle of $5 \mathrm{CB}$ we found with strong binding between LPS and protein, disruption of the interaction of 5CB with LPS will take place to a large extent, leading to a greater change in the tilt angle of $5 \mathrm{CB}$ (Fig. $7 \mathrm{H}$ ). On this basis, we can predict that the Hb-LPS interaction is the strongest, followed by BSA-LPS and least for LZM-LPS. Interestingly, this observation was also supported by (Yang and Yang, 2008) study in which the binding constants of these above mentioned interactions were calculated by using the SPR technique. The direct correlation of binding constants were obtained by using SPR along with the tilt angles and birefringence values of the LC for LPS interactions with $\mathrm{Hb}, \mathrm{BSA}$ and LZM. We also investigated whether protein driven reorientation of
LPS on the LC interface (via specific binding of the proteins to the LPS-decorated interface) was accompanied by redistribution or displacement of the LPS from the interface. Fig. 7J shows the interfacial density of protein (as inferred by the fluorescence intensity of 20 mole \% FITC-conjugated BSA at the interface) was measured to increase at the LPS laden LC interface (Fig. 7I) that was incubated against aqueous solution of protein. This increase in interfacial density of protein (BSA) suggests that the change in orientation of LCs (Fig. 7K and 7L) at LPS laden interface through protein-LPS binding affinity was driven by redistribution of LPS monolayer which further supports that the tilting of LC hints about the different binding affinity of proteins towards LPS. Overall, the results presented in this study suggested that LCs could offer not only the basis of a novel analytical tool for fundamental studies of protein-LPS interactions, but could also be used to quantify specific binding of proteins on LPS-decorated aqueous-LC interfaces.

\section{pH-Driven Ordering Transitions in Liquid Crystal Induced by Conformational Changes of Cardiolipin}

Cardiolipin (CL), being an exclusive mitochondrial inner membrane phospholipid involved in various mitochondrial functions, marked change in the polymorphic behavior of the CL is known to affect a range of cellular processes (Claypool and Koehler, 2012; Osman et al., 2011; Dowhan, 1997; Hoch, 1992; Awasti et al., 1971; Robinson, 1982; McAuley et al., 1999). Therefore, the structural behavior of the CL acts as a biomarker for understanding the clinical investigation of mitochondrial related problems (Houtkooper and Vaz, 2008). Thus, the study of the different conformers of the $\mathrm{CL}$ is potentially important for therapeutical and clinical point of view. In this study, we were interested to use the intrinsic cooperative behavior associated with the long-range orientational order of the LC phase as a probe to study the different polymorphic behaviors of CL.We investigate the interfacial phenomena occurring at aqueous-LC interfaces that trigger an orientational ordering transition of the $\mathrm{LC}$ in the presence of CL by varying $\mathrm{pH}$ (Sidiq et al., 2015).

Our first set of experiments revolves around the influence of $\mathrm{CL}$ on the orientational ordering transition 
of 5CB LCs at aqueous-LC interfaces. Interestingly, we found that unlike other lipids (such as DLPC), anchoring transition of LCs remains invariant through adsorption of $\mathrm{CL}$ at these interfaces. This made us ponder to explore other conformations $\left(\mathrm{H}_{2} \mathrm{~A}, \mathrm{HA}^{-}\right.$ and $\mathrm{A}^{2-}$ ) of the $\mathrm{CL}$ molecules in which it may couple strongly with the LC ordering at the aqueous-LC interfaces and could result in an orientational ordering transition of the LCs. As we know that CL exists in three different equilibrium species $\left(\mathrm{H}_{2} \mathrm{~A}, \mathrm{HA}^{-}\right.$and $\mathrm{A}^{2-}$ ) due to very different $\mathrm{pK}_{\mathrm{a}}$ values of the two phosphate groups (see Fig. 8A). At very low $\mathrm{pH}$ ( $\mathrm{pH}$ $<2.8$ ), the dominant species is $\mathrm{H}_{2} \mathrm{~A}$ (both the phosphate groups are protonated) and as the $\mathrm{pH}$ is gradually increased ( $\mathrm{pH} \sim 5.5)$, first cyclic/closed form $\left(\mathrm{HA}^{-}\right)$becomes predominant followed by the open conformer $\left(\mathrm{pH}>7, \mathrm{~A}^{2-}\right)$. Here, we chose three different $\mathrm{pH}$ (i.e., $\mathrm{pH}=2,4,8$ ) in which $\mathrm{CL}$ molecules adopt mostly one of the conformations at each $\mathrm{pH}$. In these experiments, the effects of three different conformational isomeric forms of the $\mathrm{CL}$ are observed to cause the response of the LC ordering to vary significantly from one to another at those interfaces. An ordering transition of the $\mathrm{LC}$ was observed when the $\mathrm{CL}$ is mostly in undissociated (at $\mathrm{pH} 2$ ) and/or in bicyclic (at $\mathrm{pH} 4$ ) conformation in which LC shows changes in the optical appearance from bright to dark (Fig. 8B and C and Fig. 8D and E). By contrast, no change in the optical appearance of the LC was observed when the $\mathrm{pH}$ of the system increases to 8 or higher in which the CL mostly exists in the open conformation (Fig. 8F and 8G). From these results, we speculate that reduction in head group-head group repulsion of the CL (due to the bicyclic form or un dissociated form) at $\mathrm{pH} 4$ and $\mathrm{pH} 2$ may be playing a role in the efficient packing of alkyl chains of the $\mathrm{CL}$ at these interfaces leading to orientational ordering transitions in LCs whereas head group-head group repulsion of the $\mathrm{CL}$ at $\mathrm{pH} 8$ in which CL exists mostly in the open form $\left(\mathrm{A}^{2-}\right)$ was unable to induce an ordering transition of $5 \mathrm{CB}$ at aqueous/LC interfaces. A detailed study was performed by varying concentration of the $\mathrm{CL}$ in a wide range of $\mathrm{pH}$ at aqueous-LC interfaces. These results also support our proposition that among the three conformational forms of the CL, the undissociated and the bicyclic conformations led to self-assembly of CL alkyl chains at aqueous-LC interfaces and thus tune the ordering transition of the LCs. In addition, with increase in undissociated form of $\mathrm{CL}$ (i.e., at lower $\mathrm{pH}$ ) the sensitivity and the response time increases toward orientational ordering transition of the $\mathrm{LC}$ at aqueousLC interfaces. This led to the high sensitivity of CL detection at LC/aqueous interface in the micromolar range $(0-10 \mu \mathrm{M})$, which lies in the physiological range of CL in mitochondrial membrane providing a simple, rapid and accessible method for the detection of CL.

Next, fluorescence measurements were performed to quantify and to determine the influence of CL density on the LC ordering transition at different $\mathrm{pH}$ conditions. Inspection of Fig. $8 \mathrm{H}$ reveals that the interface that was incubated against the solution containing BODIPY-CL at $\mathrm{pH} 7$ exhibited fluorescence intensity comparable to the control but lower than an aqueous solution of $\mathrm{CL}$ at $\mathrm{pH} 2$. This results in that $\mathrm{CL}$ adsorbed onto aqueous-LC interfaces at $\mathrm{pH} 2$ instead of $\mathrm{pH} 7$ confirming that the $\mathrm{pH}$-dependent conformational forms of CL have different abilities to self-assemble at LC-aqueous interfaces leading to ordering transition of $\mathrm{LC}$ to a different extent at different $\mathrm{pH}$ values. Further we performed another experiment in which LC-filled grid in contact with the CL $(0.3 \mathrm{mM})$ at $\mathrm{pH} 8(10 \mathrm{mM}$ tris buffer) on incubation for $90 \mathrm{~min}$ was transferred to a buffer solution (CL free) at $\mathrm{pH} 2$ (10 mM citric acid). During this transfer, we observed an immediate change in optical response of the LC from bright to dark (Fig. $8 \mathrm{I}$ and $8 \mathrm{~J}$ ) whereas no visual change was observed in the epifluorescence micrographs of the nematic $5 \mathrm{CB}$ film (containing $0.3 \mathrm{mM} \mathrm{CL}$ and $0.025 \mathrm{~mol} \%$ BODIPY-CL) at aqueous-LC interfaces (Fig. 8K and $8 \mathrm{~L})$. These observations results that the ordering transition of the LC at aqueous-LC interfaces was explicitly controlled by the change in the conformations adopted by the $\mathrm{CL}$ (at different $\mathrm{pHs}$ ) and not by the unequal interfacial surface density of the CL at different $\mathrm{pHs}$. In addition, orientational ordering transition of the LC was also observed as a function of increasing the ionic strength (buffer capacity) and strongly influenced in presence of mono and divalent cations. Specifically, we found that change in head group-head group repulsion of the central phosphatidyl groups of the CL plays a key role in tuning the lipid packing efficiency and thus responses to interfacial phenomena. 


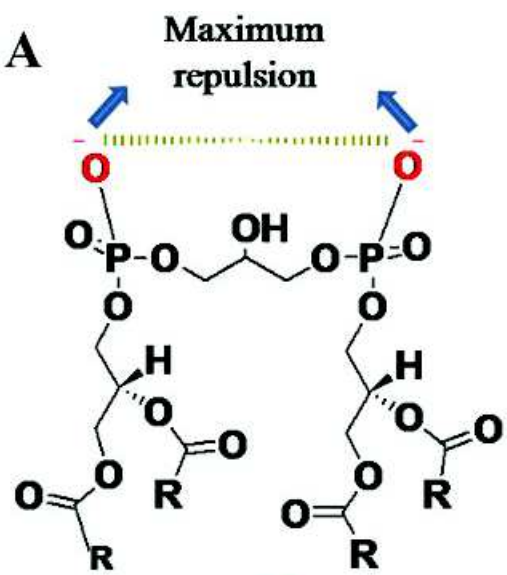

$\mathbf{A}^{2-}$

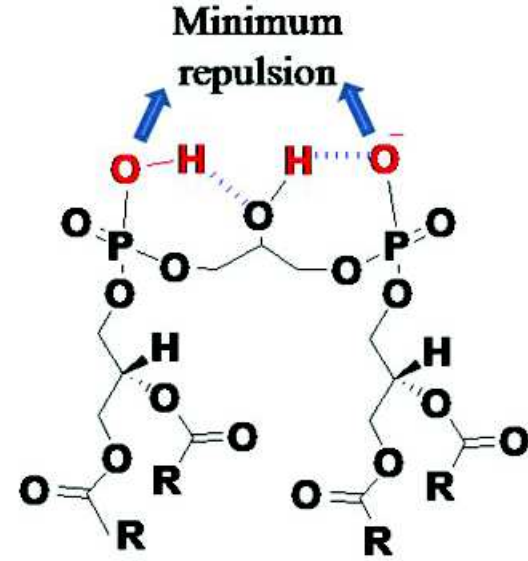

$\mathbf{H A}^{-}$

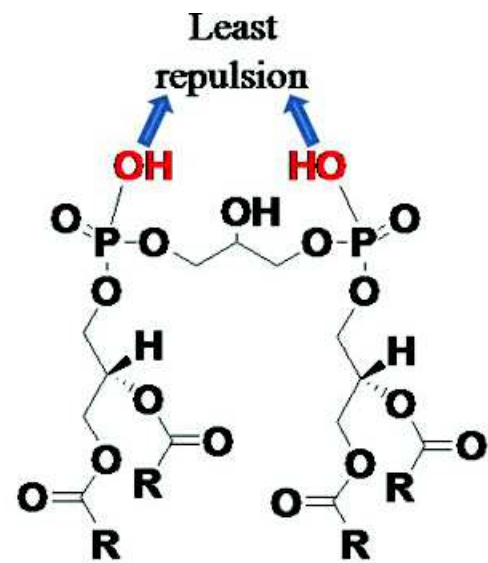

$\mathrm{H}_{2} \mathrm{~A}$
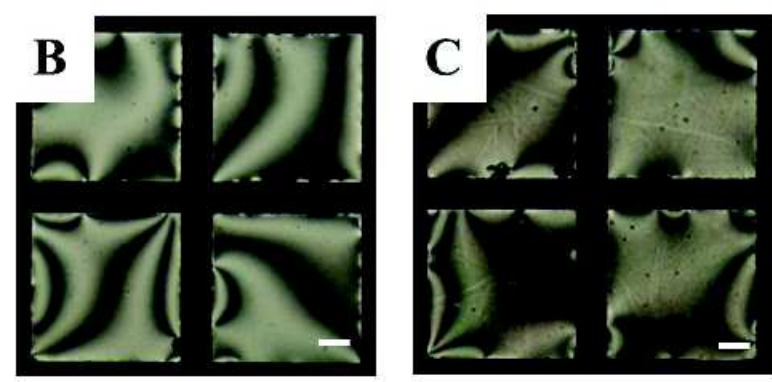

\section{$\mathbf{H}$}
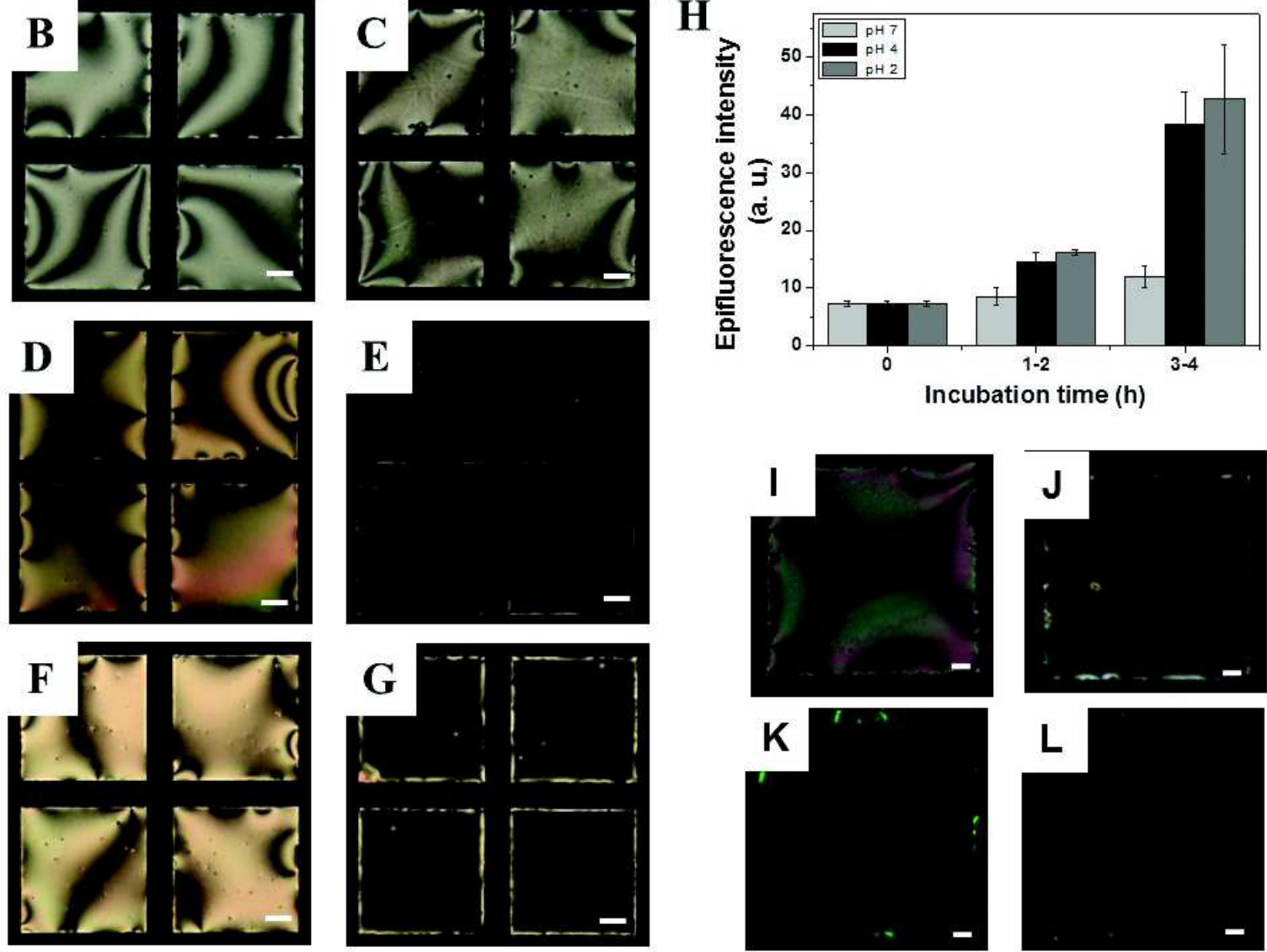

Fig. 8: (A) Molecular conformations of $\mathrm{CL}$ at different $\mathrm{pH}$ conditions. Open form, bicyclic/closed form and undissociated form. Cross-polarized optical images of $5 \mathrm{CB}$ films $(\mathrm{B})$ with tris buffer $(\mathrm{pH}=8)$, D) with citric acid buffer $(\mathrm{pH}=4)$ and $(\mathrm{F})$ with citric acid buffer $(\mathrm{pH}=2$ ). Panels $\mathrm{C}, \mathrm{E}$ and $\mathrm{G}$ represent the corresponding optical micrographs of 5CB films after addition of an aqueous solution of $0.5 \mathrm{mM}$ CL immersed in buffer of pH 8, 4 and 2, respectively. (H) Plot of epifluorescence intensity of BODIPY-CL at the aqueous-LC interface without and with $\mathrm{CL}$ vesicles against an aqueous buffer of pH 7 , 4 and 2 at different incubation periods. Optical micrographs (crossed polars) of CL laden aqueous-5CB interface following 90 min incubation against (I) an aqueous buffer of pH 8 (10 mM tris) and (J) after exchanging the same CL laden nematic 5CB film with aqueous buffer of pH $2(10 \mathrm{mM}$ citric acid). Corresponding epifluorescence micrographs of the nematic 5CB film (containing $0.3 \mathrm{mM}$ CL and $0.025 \mathrm{~mol} \%$ BODIPY-CL) are shown in panels $\mathrm{K}$ and $\mathrm{L}$, respectively. Scale bar $=40 \mu \mathrm{m}$. (Reproduced with permission from Sidiq et al., (2015)) 
Finally, Langmuir-Blodgett (LB) and polarization modulation infrared reflection absorption spectroscopy (PM-IRRAS) measurements were performed to provide further insight into the alkyl chains organizations at the molecular level occurring within a thin film of $\mathrm{CL}$ at different $\mathrm{pH}$ and ionic conditions and thus attributes different ordering transitions of the LCs. Careful inspection of the surface pressure versus area isotherms of $\mathrm{CL}$ monolayers at $\mathrm{pH} 2, \mathrm{pH}$ 8 and at $\mathrm{pH} 8$ in the presence of $\mathrm{Ca}^{2+}$ ions (Fig. 9A, 9B, 9C), reveals increased area per molecule at $\mathrm{pH} 8$ $\left(\sim 235.1 \pm 0.9 \AA^{2}\right)$ than $\mathrm{pH} 2\left(220.5 \pm 0.3 \AA^{2}\right)$ and $\mathrm{pH}$ 8 in the presence of $\mathrm{Ca}^{2+}$ ions $(216.5 \pm 0.9)$. It may be noted that in the presence of $\mathrm{Ca}^{2+}$ ions at $\mathrm{pH} 8$, the limiting area per molecule was found to be almost the same to that of $\mathrm{pH} 2$ which provide evidence that headgroup-headgroup repulsion of the CL determines the lipid packing efficiency, which largely dictates the resulting ordering transition of the LC. On the other hand, PM-IRRAS spectra showed the $\mathrm{C}-\mathrm{H}$ stretching, $\mathrm{C}=\mathrm{O}$ stretching, and asymmetric phosphate O-P-O stretching regions at $\mathrm{pH} 2$ and $\mathrm{pH} 8$ which encode information about hydrocarbon chain conformation, hydration and hydrocarbon chain packing interactions and the polarity of the phosphate polar head groups, respectively. On evaluating the methylene $\mathrm{C}-\mathrm{H}$ stretching region of PM-IRRAS spectra of CL films at different $\mathrm{pH}$ (Fig. 9D), we found that two intense bands corresponding to the asymmetric and symmetric $\mathrm{CH}_{2}$ vibrations of the lipid hydrocarbon chains that appear at 2924 and $2853 \mathrm{~cm}^{-1}$, respectively (Fig. 9D). The other peaks at 2881 and $2961 \mathrm{~cm}^{-1}$ are due to the symmetric and antisymmetric $\mathrm{CH}_{3}$ stretching modes, respectively. In contrast, at $\mathrm{pH} 8$, a significant change in the peak intensity was observed for both $-\mathrm{CH}_{3}$ and $-\mathrm{CH}_{2}$ bands. Also, these bands were found to become broadened with a change in the peak frequency at $\mathrm{pH} 8$. From the spectroscopic data we found that the absolute value of the $-\mathrm{CH}_{2}$ $\left(\mathrm{v}_{\mathrm{a}}\right)$ absorption frequencies of $\mathrm{CL}$ at $\mathrm{pH} 8$ and $\mathrm{pH} 2$ are 2926 and $2924 \mathrm{~cm}^{-1}$, respectively. However, in the presence of $\mathrm{Ca}^{2+}$ at $\mathrm{pH} 8$, peaks appear at the same position as those in the presence of $\mathrm{pH} 2$. Therefore, it may be hypothesized that at $\mathrm{pH} 8$, the packing efficiency of the CL is lower than at $\mathrm{pH} 2$ due to the formation of unoccupied inter-chain space. We also find evidence in the change in the carbonyl and $\mathrm{P}-\mathrm{O}$ vibration modes of the CL in between 1200 and $1800 \mathrm{~cm}^{-1}$ at different $\mathrm{pHs}$. A substantial increase in the peak intensity and a considerable shift to the higher wave number of both $-\mathrm{C}=\mathrm{O}$ and $\mathrm{P}-\mathrm{O}$ stretching modes at $\mathrm{pH} 8$ than $\mathrm{pH} 2$. This clearly signifies a distinct variation of the headgroup conformations of the $\mathrm{CL}$ at varying $\mathrm{pH}$. All these results indicate a conformational change in the $\mathrm{CL}$ in the interfacial region at different $\mathrm{pHs}$. We believe that this conformational change leads to buckling of the alkyl chains of the CL, which is responsible for the different orientational behavior of the 5CB LC. On aqueousLC interfaces at $\mathrm{pH} 2,5 \mathrm{CB}$ exhibits homeotropic anchoring in the presence of $\mathrm{CL}$, while on surfaces at $\mathrm{pH} 8,5 \mathrm{CB}$ exhibits planar (tilted) anchoring. Interestingly, at $\mathrm{pH} 8$, in the presence of $\mathrm{Ca}^{2+}$ ions, both peak intensity and position of different vibrational bands appear similar to that in the presence of $\mathrm{pH} 2$ (Fig. 9D). This is due to the complexation of $\mathrm{Ca}^{2+}$ ions with the negatively charged head groups of the CL that changes the molecular conformations back as it was for $\mathrm{pH} 2$ and therefore an ordering transition of LC is realized. These experiments clearly established that the change in the surface charge of the CL head group through change in $\mathrm{pH}$ or addition
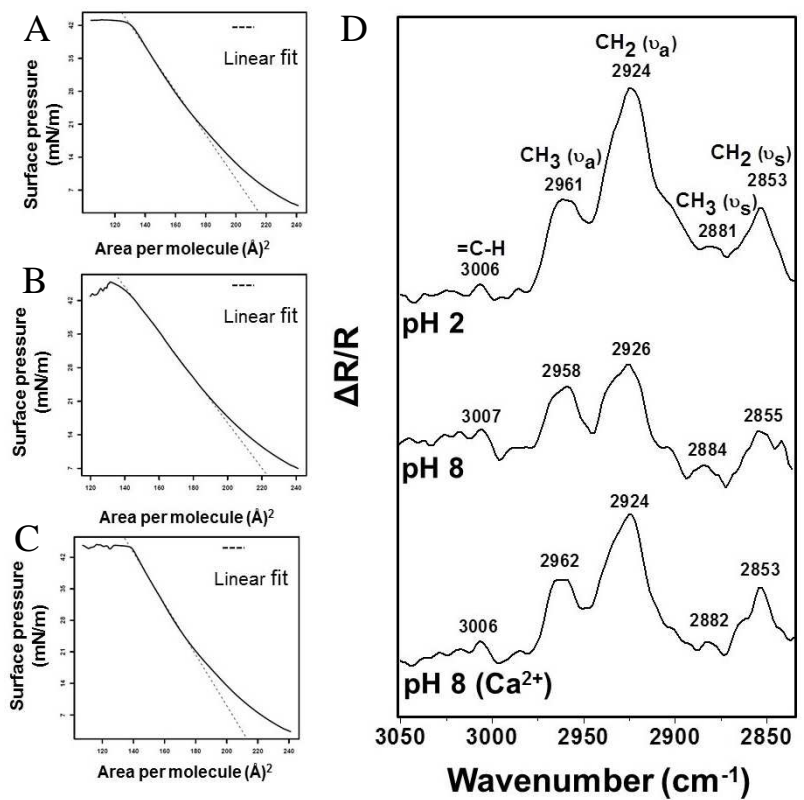

Fig. 9: Surface pressure $(\pi)$-area per molecule $\left(A_{m}\right)$ isotherm for cardiolipin on A) pH 2, B) pH 8 and C) pH 8 (5 mM $\mathrm{Ca}^{2+}$ ) based sub-phase at $25{ }^{\circ} \mathrm{C}$. D) PM-IRRAS spectra generated from alkyl region of $\mathrm{CL}$ monolayers prepared at pH 2, pH 8 and pH $8\left(\mathrm{Ca}^{2+}\right)$ onto SAM $\left(\mathrm{HS}\left(\mathrm{CH}_{2}\right)_{6} \mathrm{COOH}\right)$ supported on a uniformly deposited film of gold. (Reproduced with permission from Sidiq et al., (2015)) 
of cations causes significant conformational changes of the acyl chains of CL and thus attributes different ordering transitions of the LCs at different $\mathrm{pHs}$ at aqueous-LC interfaces. Overall, the results in our study suggest that LCs can offer a novel tool for fundamental understanding of CL-induced ordering of LC and in particular, they offer new methods to report different conformational forms of the CL at aqueous-LC interfaces.

\section{Design of Bio-Molecular Interfaces using Liquid Crystals Demonstrating Endotoxin Interactions with Bacterial Cell Wall Components}

Peptidoglycan (PG) and lipoteichoic acid (LTA), two active outer cell membrane components of bacteria, have drawn widespread interest in clinically investigating their influence over the consequence of endotoxicity induced by LPS in mammalian hosts, implying the huge importance of studying this interaction for clinical understanding associated with Gram negative bacterial infections (Wang et al., 2011; Takada and Galanos, 1987; Parant et al., 1990; Wary et al., 2001; Wolfert et al., 2002; Sugawara et al., 1999; Kusunoki et al., 1995; Nisengard and Newman, 1994). In this advance, we explore the use of the LC as novel quantitative analytical tool to report interaction of bacterial endotoxin (LPS) with PG and LTA at aqueous-LC interfaces (Das et al., 2015). The approach revolves around the formation of LPS laden aqueous-LC interface which leads to homeotropic orientation of LCs as already mentioned above. Prior to study the interactions between LPS with cell membrane components at aqueous-LC interfaces, we verified that the surface used in our study was decorated with LPS using LB, fluorescence and PM-IRRAS measurements. We exploited Langmuir film balance technique (Lin et al., 2011; Meli et al., 2008) to pre-organize monolayer of LPS molecules at the air-water interface and quantitatively transfer LPS/LPS doped with $0.2 \%$ FITC-LPS monolayers from the air-water interface to the LCaqueous interface at different surface pressures. Fig. 10 shows the polarized optical micrographs and the respective epifluorescence micrographs of LPS monolayers formed via Langmuir transfer from the air-water interface at different surface pressures. Inspection of polarized micrographs resulted that the lower density of LPS monolayers at the LC interface gave rise to planar orientation of the LC (Fig. 10A and 10B) while the higher areal density (higher surface pressure) LPS film transferred gave rise to homeotropic LC orientation as shown in Fig. 10C. Interestingly, the corresponding epifluorescence measurements (Fig. 10D-F) shows the increase in fluorescence intensity indicate the quantitative transfer of LPS from the aqueous-air interface onto the aqueous-LC interface. These results confirmed that LPS-LC interactions lead to the ordering transition of the LC at aqueous interface. Next, we utilized PMIRRAS to further characterize the adsorption of LPS on $5 \mathrm{CB}$ aqueous interface. For this, we functionalized micro-pillars (array of nickel (Ni) micro-pillars electroplated on a glass substrate) with gold of thicknesses of $2000 \AA$. These functionalized gold coated micro-pillars $(2-3 \mu \mathrm{m})$ were coated with DMOAP. The IR spectra using polarization modulation of DMOAP-coated surface shows the characteristic peaks of Si-C $\left(1265 \mathrm{~cm}^{-1}\right), \mathrm{Si}-\mathrm{O}(1177$ $\left.\mathrm{cm}^{-1}, 1110 \mathrm{~cm}^{-1}\right), \mathrm{C}-\mathrm{O}\left(1043 \mathrm{~cm}^{-1}\right)$ and $\mathrm{CH}_{3}$ and $\mathrm{CH}_{2}$ stretching $\left(2960 \mathrm{~cm}^{-1}, 2924 \mathrm{~cm}^{-1}, 2853 \mathrm{~cm}^{-1}\right)$ as shown in Fig. 10G. Next we poured the 5CB into the DMOAP-coated micro-pillars. Fig. $10 \mathrm{H}$ shows the strong absorption bands of $\mathrm{C}=\mathrm{N}\left(2218 \mathrm{~cm}^{-1}\right)$ along with aromatic C-H stretching $\left(3144 \mathrm{~cm}^{-1}, 3030\right.$ $\left.\mathrm{cm}^{-1}\right)$, aliphatic $\mathrm{CH}_{2}$ and $\mathrm{CH}_{3}$ stretching $\left(2924 \mathrm{~cm}^{-1}\right.$, $2852 \mathrm{~cm}^{-1}, 2801 \mathrm{~cm}^{-1}$ ) and $\mathrm{C}-\mathrm{H}$ bending (1474 $\mathrm{cm}^{-1}$ ). Next we incubated 5CB confined in DMOAPcoated micro-pillars with an aqueous solution of 0.1 $\mathrm{mg} \mathrm{mL} \mathrm{mL}^{-1} \mathrm{LPS}$ for $6 \mathrm{~h}$ and kept this LPS adsorbed on $5 \mathrm{CB}$ film under vacuum for complete drying. Inspection of PM-IRRAS spectrum corresponding to LPS (Fig. 10I) absorbed 5CB film, we observed amide carbonyl stretching $\left(1631 \mathrm{~cm}^{-1}\right)$, broad O-H stretching $\left(3441 \mathrm{~cm}^{-1}\right)$, amide $\mathrm{N}-\mathrm{H}$ stretching $\left(3239 \mathrm{~cm}^{-1}\right)$, sharp amide $\mathrm{N}-\mathrm{H}$ bending $\left(1556 \mathrm{~cm}^{-1}\right)$, symmetric and anti-symmetric stretching of phosphate (1138 $\left.\mathrm{cm}^{-1}, 1298 \mathrm{~cm}^{-1}\right), \mathrm{CH}_{2}$ and $\mathrm{CH}_{3}$ stretching (2983 $\left.\mathrm{cm}^{-1}, 2919 \mathrm{~cm}^{-1}, 2801 \mathrm{~cm}^{-1}\right)$ and $\mathrm{C}-\mathrm{H}$ bending (1461 $\left.\mathrm{cm}^{-1}\right)$. Overall these peaks strongly support presence of LPS (Bradenburg, 1993) over 5CB film.

To study the interactions of LPS with cell membrane components, we incubated this optical cell containing LPS solution for $2 \mathrm{~h}$. Then, we exposed this LPS laden aqueous/5CB interface in contact with different cell membrane components (PG and LTA). We observed an immediate change in optical response 

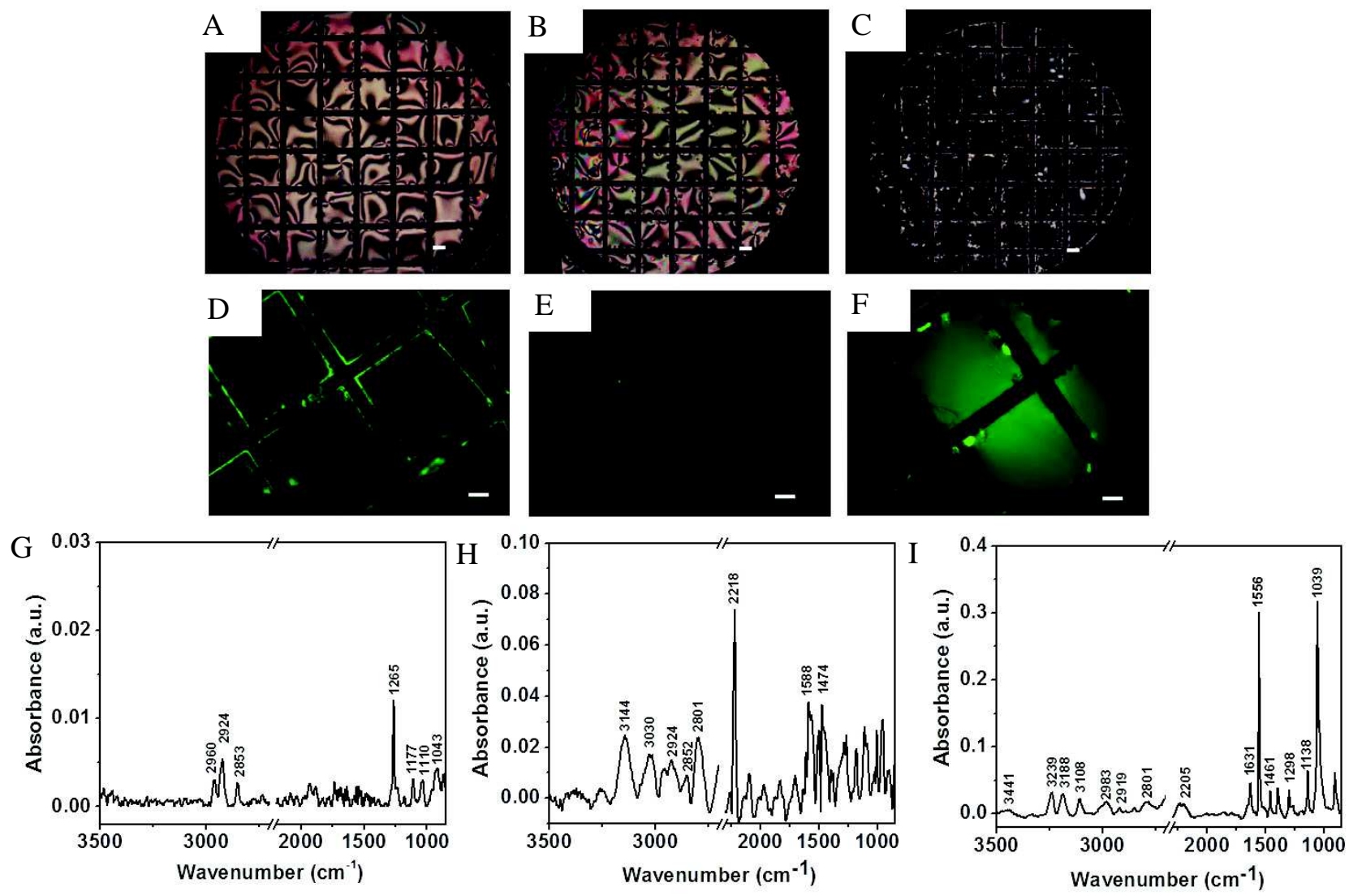

Fig. 10: Polarized light micrographs of $0.5 \%$ (mol) FITC-LPS/LPS monolayers after transfer to the LC-water interface at surface pressure of (A) 0 , (B) 30 and (C) $52 \mathrm{mN} / \mathrm{m}$. (D)-(F) Corresponding fluorescence images of films at the LC-water interface. PM-IRRAS spectra generated from (G) DMOAP, (H) 5CB and (I) LPS supported on micro-pillars-coated with a uniformly deposited film of gold. Scale bar $=40 \mu \mathrm{m}$. (Reproduced with permission from Das et al., (2015))

of the LC from dark to bright indicating an ordering transition of LCs from homeotropic to tilted state (Fig. $11 \mathrm{~A}$ and $11 \mathrm{~B}$ ) after addition of $0.1 \mathrm{mg} \mathrm{mL}^{-1}$ solution of PG. Similar change was observed after addition of $0.1 \mathrm{mg} \mathrm{mL}^{-1}$ of aqueous LTA solution onto LPS laden LC interface (Fig. 11C and 11D). This ordering transformation of LC was construed due to strong interaction between PG and LPS or LTA and LPS, which in turn disturb the ordered arrangement of LPS at aqueous-LC interface leading to the tilted orientation of LC molecules.

Next, we performed several experiments to provide insight about the interactions of PG and LTA with LPS playing a role in determining ordering transitions of LCs at these interfaces. For this, we exposed PG and LTA solutions onto LPS decorated aqueous-LC interfaces at different $\mathrm{pH}$ conditions other than physiological $\mathrm{pH}$. Interestingly, in all cases the dynamic ordering transitions towards a planar ordering in different $\mathrm{pH}$ conditions other than the physiological $\mathrm{pH}$ was found to be same. These observations, as a whole, suggest that the interactions between LPS with LTA and PG are not driven electrostatically. Another experiment we performed using aqueous starch solution. The principle motive behind performing this experiment with starch was to find out whether hydrogen bonding plays any role in the interaction of these biomolecules (PG and LTA) with LPS selfassembled at aqueous-LC interface. Vagenende $e t$ al. (2014) have shown that the self-assembly of LPS on allantoin crystals is initiated through hydrogen-bond attachment of hydrophilic LPS regions with amidegroups of allantoin. Starch is a polysaccharide based macromolecule. PG and LTA also contain sugar units, having several hydroxyl functionalities, similar to starch. Therefore, we hypothesized that, like PG and LTA, if starch also could able to induce an ordering 
transition of LPS laden interfacial 5CB, we would able to confirm that the hydrogen bonding between the polysaccharide moieties of these biomolecules (PG, LTA, starch) and LPS is mainly responsible to induce ordering transition of LPS decorated interfacial 5CB. But when we carried out this experiment, we found that the optical appearance of LPS laden 5CB interface remained dark over 2 hours of observation period after exposing $500 \mu \mathrm{g} \mathrm{mL} \mathrm{m}^{-1}$ aqueous starch solution onto $5 \mathrm{CB}$-aqueous interface. This result strongly suggests the absence of any interaction between LPS and starch at 5CB interface and hydrogen bonding between LPS and starch does not play any role in inducing ordering transition of interfacial $5 \mathrm{CB}$. Therefore, from these results we interpreted that the orientational ordering transition of the LCs likely as a result of hydrophobic interaction of PG or LTA with LPS decorated aqueous-LC interfaces.

Second, we focused to carry out investigations on the specificity of the interactions of LPS with PG and LTA, respectively. For this, we investigate the direct interaction of PG and LTA with interfacial 5CB molecules could able to alter the orientation of LCs in the absence of LPS membrane at the interface. We found that the optical appearance of LCs remained bright (even after $12 \mathrm{~h}$ of incubation or more) indicating a planar/tilted orientation of $5 \mathrm{CB}$ molecules at aqueous-LC interface. This observation clearly demonstrates that there are no direct interactions present between interfacial 5CB molecules and the cell membrane components which could perturb the orientation of LCs at these interfaces. Next, we replaced LPS with other three different phospholipids. We chose zwitter ionic DLPC and negatively charged LPA and DOPG which can form self-assembled at aqueous-LC interfaces and orient the LCs homeotropically. Interestingly, we observed that the optical appearance of LCs coupled to DLPC, LPA and DOPG decorated interfacial membrane remained dark after addition of aqueous solution of PG, LTA in Tris buffer ( $\mathrm{pH} 7.4$ ) even after $6 \mathrm{~h}$ or more incubation. This observation strongly suggests that the interaction of these cell membrane components (PG and LTA) with LPS is highly specific.

In addition to, studying the interaction of PG, LTA with LPS at these interfaces, it is very important to determine the sensitivity (Limit of detection (LOD)) of the LC based system to the realization of a novel biosensor for detection of such biomolecular interactions as the consequence of these interactions of cell membrane components with LPS in mammalian hosts is highly divergent towards the endotoxic behavior of LPS. With this idea keeping in mind, we thought to determine limit of detection (LOD) and response time of the LC based system to study biomolecular interactions. For this, we compared the dynamic response of the LCs at different concentrations of PG and LTA onto LPS decorated aqueous/LC interface through gray scale intensity and tilt angle measurements. Careful inspection of the LC ordering at different concentrations of PG and LTA, we found that the concentration of $30 \mu \mathrm{g} \mathrm{mL} \mathrm{L}^{-1}$ as LOD for PG in our LC based sensing system whereas $6 \mu \mathrm{g} \mathrm{mL}^{-1}$ is the LOD for LTA which could induce an ordering transition of the LC at LPS-laden aqueousLC interfaces (Fig. 11E). After careful observation we revealed that the birefringence colors of $5 \mathrm{CB}$ (20 $\mu \mathrm{m}$ thick film) in presence of PG onto LPS laden LC interface was distinct from that of LTA, which clearly indicates the different tilted states of 5CB molecules at aqueous-LC interfaces. Therefore, we thought that by measuring tilt angle at aqueous-LC interfaces (during LPS-PG/LTA binding events) it is possible to quantify the LC ordering at those interfaces. We observed the (for $3.5 \mathrm{~h}$ of incubation or more) maximum tilt angle of 5CB obtained for PG-LPS and LTA-LPS were $26.76 \pm 1.7$ and $38.6 \pm 2.5$, respectively, at these interfaces (Fig. 11F). Therefore, on this basis from tilt angle measurement we can say that LTA shows greater binding affinity towards LPS compared to PG. As mentioned above we have that stronger binding event could disrupt the LPS arrangement to a greater extent which, in turn, will lead to greater change in the tilt angle of $5 \mathrm{CB}$ at aqueous interfaces. In addition, our study results a continuous transition of interfacial LCs decorated with LPS has been observed in presence of PG/LTA at aqueous/LC interface. Overall, the results presented in this study suggest that LCs offer the basis of a novel analytical tool for fundamental studies of bacterial cell membrane components and endotoxin at interface and, specifically, they offer methods to quantify specific binding of PG and LTA on LPS decorated interface. 

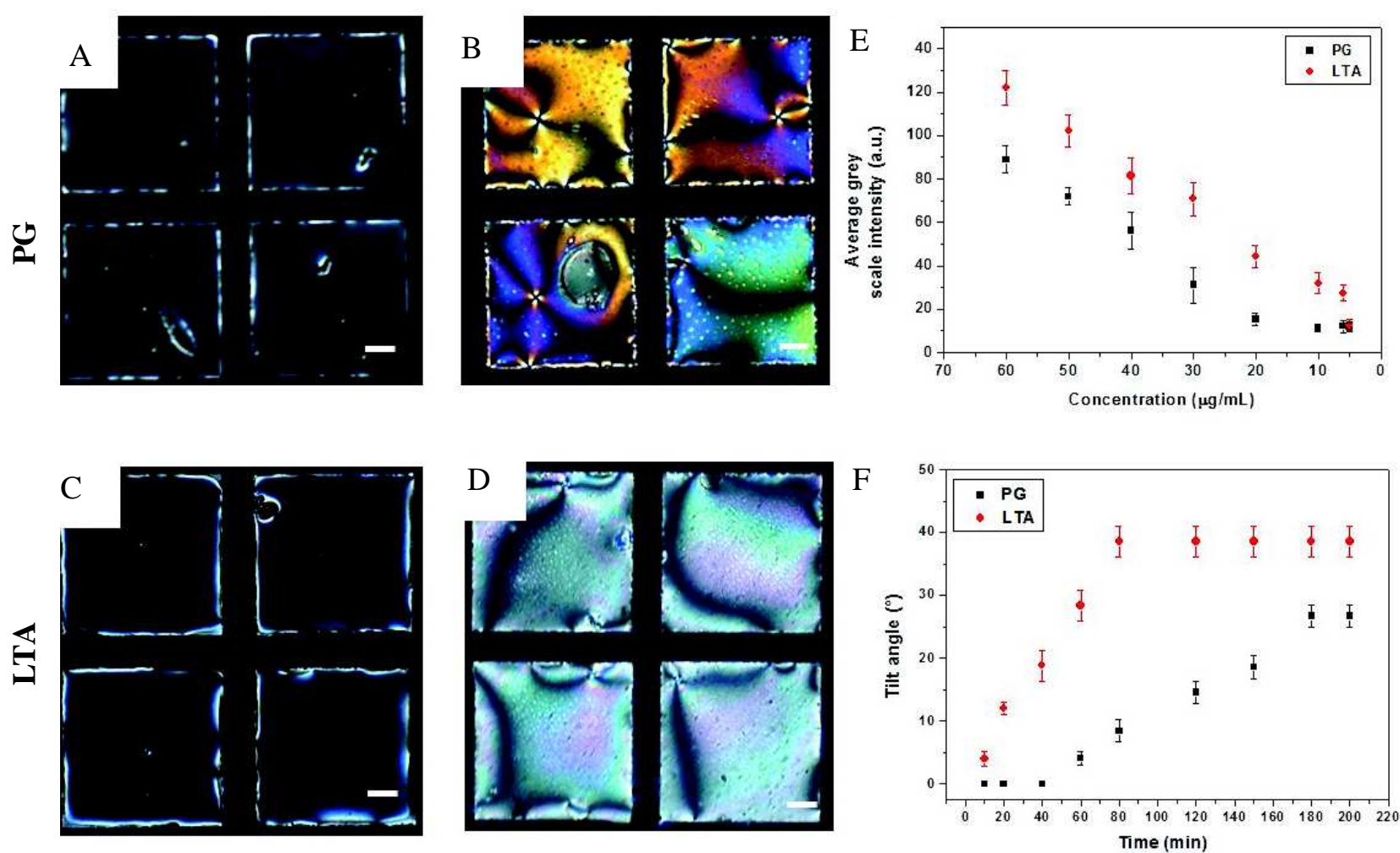

Fig. 11: Optical images (crossed polars) of 5CB hosted in gold grids supported on DMOAP-coated glass slides in contact with (A and C) LPS for $2 \mathrm{~h}$ of incubation. (B and D) after introducing aqueous PG and LTA solution onto LPS laden aqueous/ LC interface respectively. E) Represent the average gray scale intensity of optical images of 5 CB films as a function of varying concentrations of PG and LTA on LPS decorated aqueous/5CB interface. F) The tilt angle of 5CB decorated with LPS at aqueous/LC interface on exposure of $30 \mu \mathrm{g} \mathrm{mL} \mathrm{m}^{-1}$ aqueous solution of PG and LTA, respectively. Scale bar $=40 \mu \mathrm{m}$. (Reproduced with permission from Das et al., (2015))

\section{Development of New Principles for the Design of LC Based Sensors that can attach to Cell Surface}

\section{Cell-surface Sensors by Probing Poly-l-lysine (PLL)-LC Interaction}

A substantial effort has been pursued for the preparation of protein decorated solid surfaces that govern the response of LC to these protein decorated surfaces (Park and Abbott, 2008; Gupta et al., 1998; Skaife and Abbott, 2000; Skaife and Abbott, 2001; Skaife et al., 2001; Luk et al., 2003; Clare and Abbott, 2005; Jang et al., 2005; Luk et al., 2004; Govindaraju et al., 2007). However, direct protein-LC interactions in aqueous media that can mimic chemico-biological interactions remain elusive. Advances in dealing with these processes are anticipated to result from better mechanistic understanding of the interactions of proteins with LCs and provide a gateway for building fundamental in vitro and in vivo studies. In this study, we report that interaction of Poly-L-lysine (PLL) and LCs in aqueous systems could fulfill significant knowledge gap in understanding the intermolecular interactions that underlie the direct ordering of LCs with protein-decorated surfaces and hold great promise to provide LCs as cell surface sensors.

First, we investigate the orientations of nematic $5 \mathrm{CB}$ on PLL laden surface at aqueous/LC interface. Interestingly, we found that the optical appearance of LC changed from bright to dark after introduction of PLL onto 5CB/aqueous interface. The change in optical appearance of the LC is indeed consistent with adsorption and pre-organization of PLL at the interface to the aqueous phase of $5 \mathrm{CB}$, thereby allowing a rapid ordering transition. In contrast, with low molecular weight PLL solution ( $3.5 \mathrm{kDa}$ ) the optical appearance of the LC remains invariant. From these results, we hypothesized that modulation of the reactive sites of 
the PLL caused by the increase in the number of polypeptide units and the accompanying changes in overall balance of intermolecular interactions between 5CB and PLL molecules likely play an important role in governing the ordering of the LC at those interfaces. To provide additional insight into intermolecular interactions between PLL and 5CB that can be attributed to the above described ordering of the LCs, we performed Fourier transform PM-IRRAS measurements to characterize the thin films of PLL decorated with $5 \mathrm{CB}$.

Next goal involves designing a simple experimental design that exploit the use of LC droplets which are extremely attractive platforms for real time monitoring of biomolecular interactions (Manna et al., 2013; Miller et al., 2014; Tan et al., 2014; Chang and Chen, 2014; Yoon et al., 2014; Khan and Park, 2015) for the adherence on the surface of cells. In this endeavor our approach revolves around the preparation of PLL-coated LC droplets by using layerby-layer technique (see Fig. 12A). The PSS-coated LC emulsion droplets exhibited a bipolar configuration (similar to bare E7 droplets in aqueous media) whereas in presence of PLL, these droplets undergo an ordering transition from a bipolar to radial configuration as characterized by a single point defect (cross-like appearance) at the center of the droplet as characterized by using polarized optical microscopy under crossed polarand bright field, respectively. Further we explore these PLL-coated LC droplets for cell substrate interaction. Studies to exploit these modified PLL-coated LC droplets in cell based studies and other applications are currently in progress in our laboratory.

\section{Conclusion}

This review has focused on the progress that has been made in our laboratory toward the design of a range of new approaches that lead to LC materials with properties that are potentially suitable for biological applications. The first section addresses the design of LC based sensors. Although, past studies demonstrate the promise of LC interfaces for reporting biomolecular interactions, the issue of how to integrate LCs within composite materials to provide stable and robust interfaces of LCs remains an open challenge. The key result reported that CLC gels with LC-rich domains that span the thickness of the gel are stable
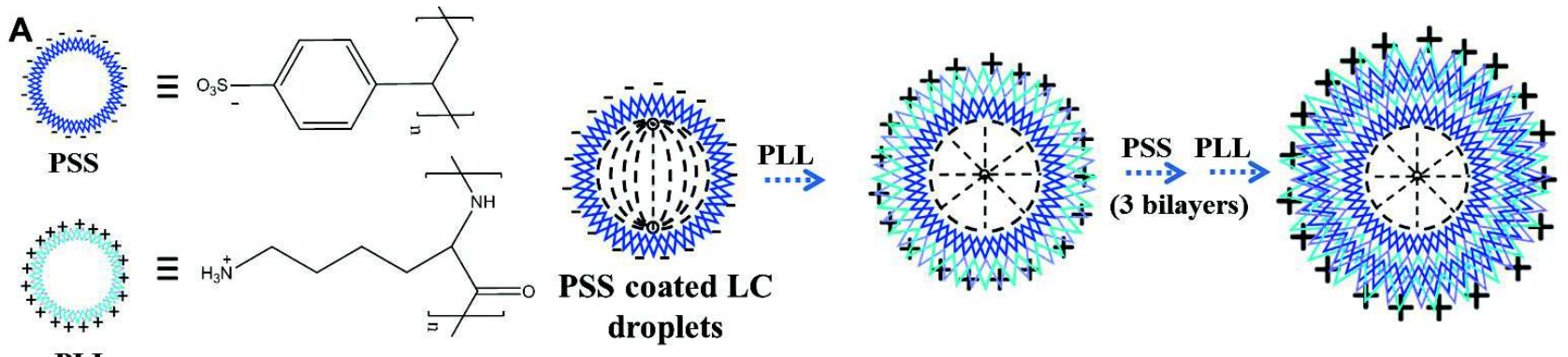

PLL

B
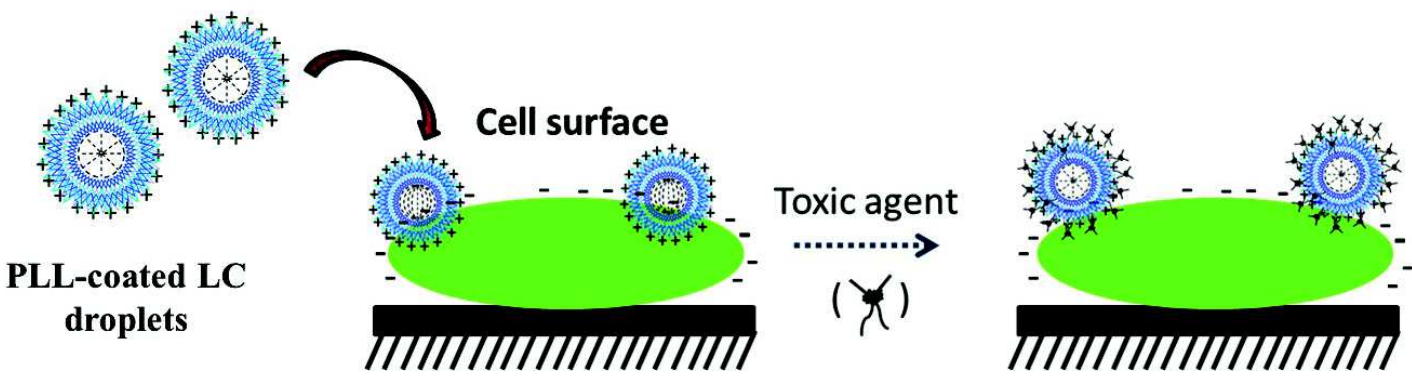

Fig. 12: A) Schematic showing the director profile of PLL/PSS multilayers adsorption on LC droplets and also the chemical structures of PSS and PLL. PSS-coated droplets exhibited a bipolar configuration with two point defects at opposite poles of the droplet surface where as in PLL radial configuration is achieved as characterized by a single point defect (cross-like appearance) at the center of the droplet. B) Schematic showing PLL-coated LC droplets anchored to cells; PLL-coated LC droplets are in bipolar states until toxic surfactant is added to promote a transition to radial states. (Sidiq et al., unpublished results) 
under water and can be used to report adsorption of biological and synthetic amphiphiles at LC interfaces as well as the processing of a substrate of an enzyme. We also observed that the response of individual domains of the LC within the CLC gel to vary significantly, suggesting that manipulation of domain size and shape may provide the basis of a general and facile method to tune the response of these LC composite materials to interfacial phenomena. Besides this, the study also establishes first to reveal direct observations of the spontaneous evolution of LC droplets with radial LC ordering in the presence of surfactants and lipids. This study affirms that ionic charge and also an additional stability mechanism (against coagulation) associated with the internal elasticity play an important role in the spontaneous formation of LC droplets with topological defects. Finally, we have shown that interactions of an enzyme with the topological defects in the LC droplets can provide means for developing new responsive soft materials.

The second broad section highlighted in the review involves the ordering of LCs at aqueous interfaces (LC-aqueous interfaces). The first part in this section reports the adsorption of biological proteins at LPS decorated aqueous-LC interfaces. We demonstrated that the LPS monolayer formed at the interface of the LC led to an anchoring transition of the LC (from homeotropic to planar) in contact with an aqueous solution of proteins (Hb, BSA and LZM). We observed, however, that protein-LPS binding affinity changed the nature of the anchoring transitions of the LC significantly; this suggested that manipulation of the interfacial phase state of the LPS monolayer (due to changes in protein-LPS binding affinities) could provide the basis for a general, facile method to tune the LPS-induced responses of the LCs to interfacial phenomena. The results also demonstrated that protein-LPS binding affinity could be quantified by measuring the optical retardation of the LC. Interestingly, the retardation values were in accordance with the order of the binding constants between LPS and different proteins; a higher binding constant resulted in a greater tilt angle of the LC. The results suggested that LCs could also be used to quantify specific binding of proteins on LPS-decorated aqueous-LC interfaces. The second part describes that the response of $\mathrm{LC}$ in the presence of $\mathrm{CL}$ at an
aqueous-LC interface is determined by the molecularlevel self-assembly of the CL. Specifically, we observed that the response of nematic $5 \mathrm{CB}$ varies significantly in the presence of different conformational forms (undissociated form, bicyclic/ closed form and open form) of the CL with varying $\mathrm{pH}$. In particular, the change in headgroup-headgroup repulsion of the central phosphatidyl groups in different conformations of the CL plays a key role in tuning the lipid packing efficiency which alters the response to interfacial phenomena. Finally, we also found that the orientational ordering of the LC is strongly influenced by the ionic strength of the buffer solution in which headgroup-headgroup repulsions of the CL can be adjusted. Our results also demonstrate that headgroup-headgroup repulsion could alter the conformation of the central glycerol and the phosphate moieties of the polar headgroup, which ultimately leads to alterations in conformation of alkyl groups. This approach provides evidence that conformational change of $\mathrm{CL}$ induces a predictable LC anchoring transition. The third part of this section report a subtle and robust LC-based sensing platform to study quantitatively the interaction of LPS with bacterial cell membrane components. We have characterized the LPS laden aqueous/5CB interface using LB technique and PM-IRRAS measurement. Strong interaction between the cell membrane components (PG, LTA) and LPS induces the orientational ordering transition of LCs at aqueous-LC interfaces through changes in the optical appearance of the LCs. These binding events are highly specific towards LPS in response to different lipids. The detection limit of our LC based system towards these biomolecular interactions was found $6 \mu \mathrm{g} \mathrm{mL} L^{-1}$ and $30 \mu \mathrm{g} \mathrm{mL}^{-1}$ for LTA-LPS and PG-LPS interactions, respectively, indicating high sensitivity of our system towards these biomolecular binding events at aqueous/LC interface. Finally, we have shown the quantitative approach of studying different binding affinities of PG and LTA towards LPS in light of tilt angle measurement.The results offer methods to quantify specific binding of PG and LTA on LPS decorated interface.

The last section addressed in this review involves cell surface sensing using LCs. The key result report the direct PLL-LC interactions in aqueous media trigger LC orientation. Studies to expand the potential utility of PLL-coated LC droplets into the realm of 
reporting cell based interactions may be used for mimic other important biological interactions.

We end by noting that examples presented in this review demonstrate new approaches for developing LC based stimuli responsive materials that are expected to improve the fundamental understanding of biomolecular interactions and provide a gateway for the further advancement in LC-based sensor designing. Finally, at the cellular level, LCs thereby opens up new unique opportunities for discovering several interfacial phenomena related to cellular interactions which ensure enormous implications for cell biology and regenerative medicine.

\section{References}

Agarwal A, Huang E, Palecek S and Abbott N L (2008) Optically Responsive and Mechanically Tunable Colloid-In-Liquid Crystal Gels that Support Growth of Fibroblasts $A d v$ Mater 20 4804-4809

Agarwal A, Sidiq S, Setia S, Bukusoglu E, de Pablo J J, Pal S K and Abbott N L (2013) Colloid-in-liquid crystal gels that respond to biomolecular interactions Small 9 2785-2795

Alino V J, Pang J and Yang K-L (2011) Liquid Crystal Droplets as a Hosting and Sensing Platform for Developing Immunoassays Langmuir 27 11784-11789

Alino V J, Tay K X, Khan S A and Yang K-L (2012) Inkjet Printing and Release of Monodisperse Liquid Crystal Droplets from Solid Surfaces Langmuir 28 14540-14546

Awasti Y C, Chuang T F, Keenan T W and Crane F L (1971) Tightly Bound Cardiolipin in Cytochrome Oxidase Biochim Biophys Acta 226 42-52

Bai Y, Abbasi R, Wang C and Abbott N L (2014) Liquid Crystals Anchored on Mixed Monolayers of Chiral versus Achiral Molecules: Continuous Change in Orientation as a Function of Enantiomeric Excess Angew Chem Int Ed $\mathbf{5 3}$ 8079-8083

Bera T and Fang J (2012) Polyelectrolyte-coated liquid crystal droplets for detecting charged macromolecules $J$ Mater Chem 22 6807-6812

Bera T and Fang J (2013) Optical Detection of Lithocholic Acid with Liquid Crystal Emulsions Langmuir 29 387-392

Brake J M and Abbott N L (2002) An Experimental System for Imaging the Reversible Adsorption of Amphiphiles at Aqueous-Liquid Crystal Interfaces Langmuir 18 61016109

\section{Acknowledgement}

This work was carried out with the financial support from IISER Mohali and Department of Atomic Energy (DAE-BRNS) bearing sanction No. 2012/20/ 34/7/BRNS. We thank different journals who permitted use of full articles (including figures) reproduced in this review article. S Sidiq acknowledges the receipt of a graduate fellowship from the U. G. C., New Delhi. Dr. SK Pal is grateful for INSA Medal for Young Scientist 2015 and the financial support from INSA bearing Sanction No. SP/YSP/124/2015/433.

Brake J M, Daschner M K, Luk Y-Y and Abbott N L (2003) Biomolecular Interactions at Phospholipid-Decorated Surfaces of Liquid Crystals Science 302 2094-2097

Brake J M, Daschner M K and Abbott N L (2005) Formation and Characterization of Phospholipid Monolayers Spontaneously Assembled at Interfaces between Aqueous Phases and Thermotropic Liquid Crystals Langmuir 21 2218-2228

Brake J M, Mezera AD and Abbott N L (2003) Effect of Surfactant Structure on the Orientation of Liquid Crystals at AqueousLiquid Crystal Interfaces Langmuir 19 6436-6442

Brandenburg K, Koch M H J and Seydel U (1998) Biophysical characterisation of lysozyme binding to LPS Re and lipid A Eur J Biochem 258 686-695

Bradenburg K (1993) Fourier transform infrared spectroscopy characterization of the lamellar and nonlamellar structures of free lipid A and Re lipopolysaccharides from Salmonella minnesota and Escherichia coli Biophys J 64 1225-1231

Cadwell K D, Alf M E and Abbott N L (2006) Infrared Spectroscopy of Competitive Interactions between Liquid Crystals, Metal Salts, and Dimethyl Methylphosphonate at Surfaces J Phys Chem B 110 26081-26088

Carlton R J, Hunter J T, Miller D S, Abbasi R, Mushenheim P C, Tan L N and Abbott N L (2013) Chemical and biological sensing using liquid crystals Liq Cryst Rev $129-51$

Chang C-Y and Chen C-H (2014) Oligopeptide-decorated liquid crystal droplets for detecting proteases Chem Commun 50 12162/1-12162/5

Clare B H and Abbott N L (2005) Orientations of nematic liquid crystals on surfaces presenting controlled densities of 
peptides: amplification of protein-peptide binding events Langmuir 21 6451-6461

Clare B H, Guzman O, de Pablo J J and Abbott N L (2006) Measurement of the azimuthal anchoring energy of liquid crystals in contact with oligo (ethylene glycol)-terminated self-assembled monolayers supported on obliquely deposited gold films Langmuir 22 4654-4659

Claypool S M and Koehler C M (2012) The complexity of cardiolipin in health and disease Trends Biochem Sci 37 $32-41$

Clegg P S, Birgeneau R J, Park S, Garland C W, Iannacchione G S, Lehency R L and Neubert M E (2003) High-resolution Xray study of the nematic-smectic-A and smectic-Asmectic-C transitions in liquid-crystal-aerosil gels Phys Rev E Stat Nonlin Soft Matter Phys 68 031706/1-031706/7

Collings P J and Hird M (1997) Introduction to liquid crystals chemistry and physics; Taylor \& Francis: London

Das D, Sidiq S and Pal S K (2015) A simple quantitative method to study protein-lipopolysaccharide interactions by using liquid crystals ChemPhysChem 16 753-760

Das D, Sidiq S and Pal S K (2015) Design of bio-molecular interfaces using liquid crystals demonstrating endotoxin interactions with bacterial cell wall components RSC Adv 5 66476-66486

deGennes P G and Prost J (1994) The physics of liquid crystals; Oxford University Press: London

Dowhan W (1997) Molecular basis for membrane phospholipid diversity: why are there so many lipids? Annu Rev Biochem 66 199-232

Frey B L and Corn R M (1996) Covalent attachment and derivatization of poly(L-lysine) monolayers on gold surfaces as characterized by polarization-modulation FTIR spectroscopy Anal Chem 68 3187-3193

Govindaraju T, Bertics P J, Raines R T and Abbott N L (2007) Using measurements of anchoring energies of liquid crystals on surfaces to quantify proteins captured by immobilized ligands J Am Chem Soc 129 11223-11231

Gupta J K, Sivakumar S, Caruso F and Abbott N L (2009) Sizedependent ordering of liquid crystals observed in polymeric capsules with micrometer and smaller diameters Angew Chem Int Ed 48 1652-1655

Gupta V K, Skaife J J, Dubrovsky T B and Abbott N L (1998) Optical amplification of ligand-receptor binding using liquid crystals Science 279 2077-2080

Gupta J K, Zimmerman J S, de Pablo J J, Caruso F and Abbott N L (2009) Characterization of adsorbate-induced ordering transitions of liquid crystals within monodisperse droplets Langmuir 25 9016-9024

Hartono D, Xue C-Y, Yang K-L and Lanry Yung L-Y (2009) Decorating liquid crystal surfaces with proteins for realtime detection of specific protein-protein binding Adv Funct Mater 19 3574-3579

Harrison D and Fisch M R (2000) Robust liquid crystal droplets Liq Cryst 27 737-742

Hoch F L (1992) Cardiolipins and biomembrane function Biochim Biophys Acta 1113 71-133

Houtkooper R H and Vaz F M (2008) Cardiolipin, the heart of mitochondria metabolism Cell Mol Life Sci 65 2493-2506

Hu Q-Z and Jang C H (2012) Imaging trypsin activity through changes in the orientation of liquid crystals coupled to the interactions between a polyelectrolyte and a phospholipid layer ACS Appl Mater Interfaces 4 1791-1795

Hu Q Z and Jang C H (2012) Using liquid crystals to report molecular interactions between cationic antimicrobial peptides and lipid membranes Analyst 137 567-570

Jang C-H, Tingey M L, Korpi N L, Wiepz G J, Schiller J H, Bertics P J and Abbott N L (2005) Using liquid crystals to report membrane proteins captured by affinity micro contact printing from cell lysates and membrane extracts $J$ Am Chem Soc 127 8912-8913

Jerome B (1991) Surface effects and anchoring in liquid crystals Rep Prog Phys $\mathbf{5 4} 391-451$

Jurgens G, Muller M, Koch M H J and Brandenburg K (2001) Interaction of hemoglobin with enterobacterial lipopolysaccharide and lipid A Eur J Biochem 268 42334242

Khan W, Choi J H, Kim G M and Park S-Y (2011) Microfluidic formation of $\mathrm{pH}$ responsive $5 \mathrm{CB}$ droplets decorated with PAA-b-LCP Lab Chip 11 3493-3498

Khan M and Park S-Y (2014) Liquid Crystal-Based Proton Sensitive Glucose Biosensor Anal Chem 86 1493-1501

Khan M and Park S-Y (2015) Specific detection of avidin-biotin binding using liquid crystal droplets Colloid Surf B 127 241-246

Kinsinger M I, Buck M E, Abbott N L and Lynn D M (2010) Immobilization of polymer-decorated liquid crystal droplets on chemically tailored surfaces Langmuir $\mathbf{2 6}$ 10234-10242

Kusunoki T, Hailman E, Juan T S-C, Lichenstein H S and Wright S D (1995) Molecules from Staphylococcus aureus that bind CD14 and stimulate innate immune responses $J$ Exp 


\section{Med 182 1673-1682}

Lavrentovich O D (1998) Topological defects in dispersed words and worlds around liquid crystals, or liquid crystal drops Liq Cryst 24 117-126

Lin I H, Miller D S, Bertics P J, Murphy C J, de Pablo J J and Abbott N L (2011) Endotoxin-Induced Structural Transformations in Liquid Crystalline Droplets Science 332 1297-1300

Liu D, Hu Q Z and Jang C H (2013) Orientational Behaviors of Liquid Crystals Coupled to Chitosan-Disrupted Phospholipid Membranes at the Aqueous-Liquid Crystal Interface Colloids Surf B 108 142-146

Lockwood N A, de Pablo J J and Abbott N L (2005) Influence of Surfactant Tail Branching and Organization on the Orientation of Liquid Crystals at Aqueous-Liquid Crystal Interfaces Langmuir 21 6805-6814

Lockwood N A, Mohr J C, Ji L, Murphy C J, Palecek S R, de Pablo J J and Abbott N L (2006) Thermotropic Liquid Crystals as Substrates for Imaging the Reorganization of Matrigel by Human Embryonic Stem Cells Adv Funct Mater 16 618-624

Lowe A M and Abbott N L (2012) Liquid Crystalline Materials for Biological Applications Chem Mater 24 746-758

Luk Y-Y, Tingey M L, Dickson K A, Raines R T and Abbott N L (2004) Imaging the Binding Ability of Proteins Immobilized on Surfaces with Different Orientations by Using Liquid Crystals J Am Chem Soc 126 9024-9032

Luk Y-Y, Tingey M L, Hall D J, Israel B A, Murphy C J, Bertics P J and Abbott N L (2003) Using Liquid Crystals to Amplify Protein-Receptor Interactions: Design of Surfaces with Nanometer-Scale Topography that Present HistidineTagged Protein Receptors Langmuir 19 1671-1680

Machnicki M, Zimecki M and Zagulski T (1993) Lactoferrin regulates the release of tumour necrosis factor alpha and interleukin 6 in vivo Int J Exp Pathol 74 433-439

Manna U, Zayas-Gonzalez Y M, Carlton R J, Caruso F, Abbott N L and Lynn D M (2013) Liquid Crystal Chemical Sensors That Cells Can Wear Angew Chem Int Ed 52 1401114015

McAuley K E, Fyfe P K, Ridge J P, Isaacs N W, Cogdell R J and Jones M R (1999) Structural Details of an Interaction between Cardiolipin and an Integral Membrane Protein Proc Natl Acad Sci USA 96 14706-14711

Meli M V, Lin I H and Abbott N L (2008) Preparation of Microscopic and Planar Oil-Water Interfaces That Are Decorated with Prescribed Densities of Insoluble
Amphiphiles J Am Chem Soc 130 4326-4333

Miller D S and Abbott N L (2013) Influence of droplet size, pH and ionic strength on endotoxin-triggered ordering transitions in liquid crystalline droplets Soft Matter $9374-$ 382

Miller D S, Wang X and Abbott N L (2014) Design of Functional Materials Based on Liquid Crystalline Droplets Chem Mater 26 496-506

Munir S, Khan M and Park S-Y (2015) Bienzyme liquid-crystalbased cholesterol biosensor Sensors Actuat B-Chem 220 508-515

Nisengard R J and Newman M G (1994) Oral microbiology and immunology $2^{\text {nd }}$ edn $360-384$, The W.B. Saunders Co Philadelphia Pa, USA

Ohno N and Morrison D C (1989) Lipopolysaccharide interactions with lysozyme differentially affect lipopolysaccharide immunostimulatory activity $J$ Biol Chem 264 4434-4441

Osman C, Voelker D R and Langer T (2011) Making Heads or Tails of Phospholipids in Mitochondria J Cell Biol 192 716

Pal S K, Agarwal A and Abbott N L (2009) Chemically responsive gels prepared from microspheres dispersed in liquid crystals Small 5 2589-2596

Parant M, Parant F, Vinit M A, Jupin C, Noso Y and Shedid L (1990) Priming effect of muramyl peptides for induction by lipopolysaccharide of tumor necrosis factor production in mice J Leukocyte Biol 47 164-169

Park J-S and Abbott N L (2008) Ordering Transitions in Thermotropic Liquid Crystals Induced by the Interfacial Assembly and Enzymatic Processing of Oligopeptide Amphiphiles Adv Mater 20 1185-1190

Price AD and Schwartz D K (2008) DNA Hybridization-Induced Reorientation of Liquid Crystal Anchoring at the Nematic Liquid Crystal/Aqueous Interface J Am Chem Soc 130 8188-8194

Prischepa O O, Shabanov A V and Zyryanov V Y (2005) Director Configurations within Nematic Droplets Doped by Lecithin Mol Cryst Liq Cryst 438 141/[1705]-150/[1714]

Robinson N C (1982) Specificity and Binding Affinity of Phospholipids to the High-Affinity Cardiolipin Sites of Beef Heart Cytochrome c Oxidase Biochemistry 21 184188

Roth R I and Kaca W (1994) Toxicity of Hemoglobin Solutions: Hemoglobin is a Lipopolysaccharide (Lps) Binding Protein which Enhances Lps Biological Activity Biomater Artif 
Cells Immobilization Biotechnol 22 387-398

Rozenberg M and Shoham G (2007) FTIR spectra of solid poly1-lysine in the stretching NH mode range Biophys Chem 125 166-171

Sadati M, Apik A I, Armas-Perez J C, Martinez-Gonzalez J, Hernandez-Ortiz J P, Abbott N L and de Pablo J J (2015) Liquid crystal enabled early stage detection of beta amyloid formation on lipid monolayers Adv Funct Mater 25 60506060

Sidiq S, Das D and Pal S K (2014) A new pathway for the formation of radial nematic droplets within a lipid-laden aqueous-liquid crystal interface $R S C A d v 4$ 18889-18893

Sidiq S, Verma I and Pal S K (2015) pH-driven ordering transitions in liquid crystal induced by conformational changes of cardiolipin Langmuir 31 4741-4751

Sivakumar S, Wark K L, Gupta J K, Abbott N L and Caruso $\mathrm{F}(2009)$ Liquid crystal emulsions as the basis of biological sensors for the optical detection of bacteria and viruses Adv Funct Mater 19 2260-2265

Skaife J J and Abbott N L (2000) Quantitative interpretation of the optical textures of liquid crystals caused by specific binding of immunoglobulins to surface-bound antigens Langmuir 16 3529-3536

Skaife J J and Abbott N L (2001) Influence of Molecular-Level Interactions on the Orientations of Liquid Crystals Supported on Nanostructured Surfaces Presenting Specifically Bound Proteins Langmuir 17 5595-5604

Skaife J J, Brake J M and Abbott N L (2001) Influence of Nanometer-Scale Topography of Surfaces on the Orientational Response of Liquid Crystals to Proteins Specifically Bound to Surface-Immobilized Receptors Langmuir 17 5448-5457

Stewart G T (2003) Liquid crystals in biology. I. Historical, biological and medical aspects Liq Cryst 30 541-557

Stewart G T (2004) Liquid crystals in biology. II. Origins and processes of life Liq Cryst 31 443-471

Sugawara S, Arakaki R, Rikiishi H and Takada H (1999) Lipoteichoic Acid Acts as an Antagonist and an Agonist of Lipopolysaccharide on Human Gingival Fibroblasts and Monocytes in a CD14-Dependent Manner Infect Immun 67 1623-1632

Takada H and Galanos C (1987) Enhancement of endotoxin lethality and generation of anaphylactoid reactions by lipopolysaccharides in Muramyl-Dipeptide-Treated mice Infect Immun $\mathbf{5 5}$ 409-413

Takayama K, Din Z Z, Mukerjee P, Cooke P H, Kirkl and T N
(1990) Physicochemical properties of the lipopolysaccharide unit that activates B lymphocytes $J$ Biol Chem 265 14023-14029

Tan L N, Orler V J and Abbott N L (2012) Ordering transitions triggered by specific binding of vesicles to proteindecorated interfaces of thermotropic liquid crystals Langmuir 28 6364-6376

Tan L N, Wiepz G J, Miller D S, Shusta E V and Abbott N L (2014) Liquid crystal droplet-based amplification of microvesicles that are shed by mammalian cells Analyst 139 2386-2396

Terentjev E M (1995) Stability of liquid crystalline macroemulsions Europhys Lett 32 607-612

Tesh V L and Morrison D C (1988) The physical-chemical characterization and biologic activity of serum released lipopolysaccharides J Immunol 141 3523-3531

Tong X, Chung J W, Park S Y and Zhao Y (2009) Self-assembled liquid-crystal gels in an emulsion Langmuir 25 8532-8537

Vagenende V, Ching T-J, Chua R-J, Jiang Q Z and Gagnon P (2014) Self-assembly of lipopolysaccharide layers on allantoin crystals Colloids Surf B 120 8-14

Wang Y, Hu Q, Guo Y and Yu L (2015) A cationic surfactantdecorated liquid crystal sensing platform for simple and sensitive detection of acetylcholinesterase and its inhibitor Biosens Bioelectron 72 25-30

Wang J E, Jorgensen PF, Ellingsen E A, Almiof M, Theimermann C, Foster S J, Aasen A O and Solberg R (2011) Peptidoglycan primers for LPS-induced release of proinflammatory cytokines in whole human blood Shock 16 178-182

Wary G M, Foster S J, Hinds C J and Theimermann C A (2001) A cell wall component from pathogenic and non-pathogenic gram-positive bacteria (Peptidoglycan) synergises with endotoxin to cause the release of tumour necrosis factor[alpha], nitric oxide production, shock, and multiple organ injury/dysfunction in the rat Shock 15 135-142

Wolfert M A, Murray T F, Boons G J and Moore J N (2002) The origin of the synergistic effect of muramyl dipeptide with endotoxin and peptidoglycan J Biol Chem 277 3917939186

Wood T A, Lintuvuori J S, Schofield A B, Marenduzzo D and Poon W C K (2011) A self-quenched defect glass in a colloid-nematic liquid crystal composite Science 334 7983

Yang F and Yang X (2008) Kinetic analysis of interaction between lipopolysaccharide and biomolecules Front Chem China 3 14-17 
Yang Z and Abbott N L (2010) Spontaneous formation of water droplets at oil-solid interfaces Langmuir 26 13797-13804

Yoon S H, Gupta K C, Borah J S, Park S-Y, Kim Y-K, Lee J-H and Kang I-K (2014) Folate ligand anchored liquid crystal microdroplets emulsion for in vitro detection of $\mathrm{KB}$ cancer cells Langmuir 30 10668-10677

Yu B and Wright S D (1996) Catalytic properties of lipopolysaccharide (LPS) binding protein transfer of LPS to soluble CD14 J Biol Chem 271 4100-4105

Zou J, Bera T, Davis A A, Liang W and Fang J (2011) Director configuration transitions of polyelectrolyte coated liquidcrystal droplets J Phys Chem B 115 8970-8974

Zhu Q and Yang K-L (2015) Microfluidic immunoassay with plug-in liquid crystal for optical detection of antibody Anal Chim Acta 853 696-701. 